ARTICLE

\title{
Coupling of H3K27me3 recognition with transcriptional repression through the BAH-PHD-CPL2 complex in Arabidopsis
}

Yi-Zhe Zhang (1) 1,2,6, Jianlong Yuan (1) 1,2,6, Lingrui Zhang ${ }^{3,6}$, Chunxiang Chen', Yuhua Wang ${ }^{1}$, Guiping Zhang1,

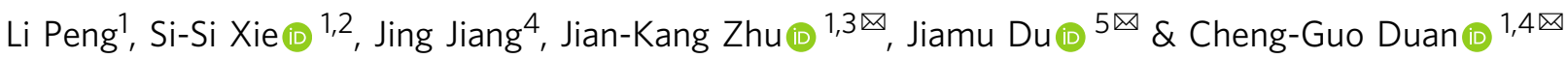

Histone 3 Lys 27 trimethylation (H3K27me3)-mediated epigenetic silencing plays a critical role in multiple biological processes. However, the H3K27me3 recognition and transcriptional repression mechanisms are only partially understood. Here, we report a mechanism for H3K27me3 recognition and transcriptional repression. Our structural and biochemical data showed that the BAH domain protein AIPP3 and the PHD proteins AIPP2 and PAIPP2 cooperate to read H3K27me3 and unmodified H3K4 histone marks, respectively, in Arabidopsis. The BAH-PHD bivalent histone reader complex silences a substantial subset of H3K27me3-enriched loci, including a number of development and stress response-related genes such as the RNA silencing effector gene ARGONAUTE 5 (AGO5). We found that the BAH-PHD module associates with CPL2, a plant-specific Pol II carboxyl terminal domain (CTD) phosphatase, to form the BAH-PHD-CPL2 complex (BPC) for transcriptional repression. The BPC complex represses transcription through CPL2-mediated CTD dephosphorylation, thereby causing inhibition of Pol II release from the transcriptional start site. Our work reveals a mechanism coupling H3K27me3 recognition with transcriptional repression through the alteration of Pol II phosphorylation states, thereby contributing to our understanding of the mechanism of H3K27me3-dependent silencing.

\footnotetext{
${ }^{1}$ Shanghai Center for Plant Stress Biology and CAS Center for Excellence in Molecular Plant Sciences, Chinese Academy of Sciences, 201602 Shanghai, China. ${ }^{2}$ University of Chinese Academy of Sciences, 100049 Beijing, China. ${ }^{3}$ Department of Horticulture and Landscape Architecture, Purdue University, West Lafayette, IN 47907, USA. ${ }^{4}$ State Key Laboratory of Crop Stress Adaptation and Improvement, School of Life Sciences, Henan University, 475004 Kaifeng, China. ${ }^{5}$ Key Laboratory of Molecular Design for Plant Cell Factory of Guangdong Higher Education Institutes, Institute of Plant and Food Science, School of Life Sciences, Southern University of Science and Technology, 518055 Shenzhen, China. ${ }^{6}$ These authors contributed equally: Yi-Zhe Zhang,

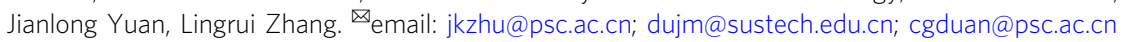


$\mathrm{n}$ eukaryotic cells, the N-terminal histone tails undergo numerous posttranslational modifications (PTMs). Histone modification acts as a mark to specify the chromatin status, as well as potential functional indications ${ }^{1}$. The deposition, recognition, and removal of specific histone PTMs are dynamically regulated by different proteins or protein complexes called "writer", "reader", and "eraser" modules, respectively ${ }^{2-4}$. The reader module can specifically recognize certain histone mark in both sequence- and modification-specific manners, and subsequently transmits the signal to downstream effectors. As a repressive epigenetic mark localized in euchromatin, deposition of trimethylation on histone $\mathrm{H} 3$ lysine 27 (H3K27me3) has been observed in many important functional genes 5 . The polycomb repressive complexes (PRCs), consisting of a different polycomb group (PcG) of proteins, have been shown to be involved in the deposition and downstream action of H3K27me3 mark ${ }^{6}$. Different PcG proteins associate to form two functionally distinct complexes, PRC1 and PRC2. The PRC1 complex has E3 ligase activity which has been shown to catalyze the monoubiquitination of histone $\mathrm{H} 2 \mathrm{~A}$ at lysine (H2Aub1), and the PRC2 complex catalyzes $\mathrm{H} 3 \mathrm{~K} 27 \mathrm{me} 2$ and $\mathrm{H} 3 \mathrm{~K} 27 \mathrm{me} 3$ (refs. ${ }^{7-10}$ ). Three major models have been proposed to explain the mechanisms of PRC complex-mediated transcription repression ${ }^{8}$. For those bivalent promoters marked by both H3K27me3 and H3K4me3 marks, PcG complexes are believed to hold the poised Pol II at the transcription start site (TSS), resulting in the inhibition of Pol II release. Alternatively, $\mathrm{PcG}$ complexes can alter the chromatin environment by inducing chromatin condensation, thereby blocking the accessibility of chromatin remodeling complexes that is required for transcription activation ${ }^{11-13}$. Third, the histone PTMs might directly prevent Pol II processivity during transcription elongation ${ }^{8}$. For example, studies in Drosophila have indicated that $\mathrm{H} 3 \mathrm{~K} 27 \mathrm{me} 3$ could limit Pol II recruitment to gene promoters ${ }^{14}$. H2Aub1 has been implicated in restraining Pol II elongation ${ }^{15,16}$. However, the detailed mechanisms through which $\mathrm{H} 3 \mathrm{~K} 27 \mathrm{me} 3$ reading is connected to transcriptional repression are not fully understood.

Histone mark recognition in plants is generally similar to that in animals, but sometimes possesses plant-specific mark-reader pairs ${ }^{17}$. The PHD and BAH domains are two types of histonebinding domains in eukaryotes ${ }^{18,19}$. The PHD finger has been reported to recognize methylated/unmethylated H3K4 marks and lysine acetylation marks ${ }^{19}$, and the $\mathrm{BAH}$ domain can bind distinct histone marks, including H3K9me2 (ref. ${ }^{20}$ ), H4K20me2 (ref. ${ }^{21}$ ), unmodified H3K4 (ref. ${ }^{22}$ ), nucleosome core particle ${ }^{23-26}$, and the more recently identified $\mathrm{H} 3 \mathrm{~K} 27 \mathrm{me} 3$ (ref. ${ }^{27-30}$ ). In plants and animals, a large number of development and environmental response-related processes are subjected to H3K27me3dependent regulation. Among them, flowering control has been a paradigmatic model for PRC complexes-mediated transcriptional repression in plants. The $\mathrm{H} 3 \mathrm{~K} 27 \mathrm{me} 3$ dynamics in the flowering repressor gene FLOWERING LOCUS C (FLC) and the florigen gene FLOWERING LOCUS T (FT) play essential roles in the flowering time control ${ }^{31}$, and the $\mathrm{H} 3 \mathrm{~K} 27 \mathrm{me} 3$ regulators influence flowering time in different ways ${ }^{27-29,32-40}$. Here, we demonstrated that the $\mathrm{BAH}$ domain-containing protein antisilencing 1 (ASI1)-IMMUNOPRECIPITATED PROTEIN 3 (AIPP3) and two PHD domain-containing proteins AIPP2/ PARALOG OF AIPP2 (PAIPP2) could form a BAH-PHD module to read $\mathrm{H} 3 \mathrm{~K} 27 \mathrm{me} 3$ and unmethylated $\mathrm{H} 3 \mathrm{~K} 4$, respectively, and coordinate in implementing transcriptional repression of hundreds of genes, particularly those development and stressresponsive genes in Arabidopsis, such as the florigen gene FT and the RNA silencing effector gene AGO5. Moreover, our structural and biochemical studies further revealed the molecular basis for the specific recognition of these histone marks. We also revealed that the BAH-PHD module represses the release of Pol II from TSS regions by cooperating with CPL2, a known plant-specific Pol II carboxyl terminal domain (CTD)-Ser5 phosphatase. Collectively, our findings reveal a coupling of the H3K27me3 recognition and downstream transcriptional repression through the BPC complex. This pathway may represent a mechanism of H3K27me3-mediated gene silencing.

\section{Results}

BAH protein AIPP3 associates with PHD proteins and CPL2 to form a protein complex in Arabidopsis. Through a mass spectrometry (MS) analysis of the chromatin regulator ASI1, we previously demonstrated that $\mathrm{BAH}$ domain-containing protein AIPP3, and PHD protein AIPP2 and CPL2 are associated with ASI1 (refs. ${ }^{41,42}$ ). AIPP2 is known to interact with AIPP3 and CPL2 (ref. ${ }^{41}$ ). This association was further confirmed by immunoprecipitation assays coupled to a mass spectrometry analysis (IP-MS) of AIPP3, AIPP2, and CPL2 in which AIPP3, AIPP2, and CPL2 could be mutually co-purified with one another except for ASI1 (Fig. 1a and Supplementary Data 1). Interestingly, another PHD protein encoded by AT5G16680 (Supplementary Fig. 1), the closest paralog of AIPP2 (hereafter referred to as PAIPP2) in Arabidopsis, was co-purified with AIPP3 and CPL2, and AIPP3 and CPL2 were also present in the IP-MS of PAIPP2 (Fig. 1a). The yeast two-hybrid (Y2H) and split luciferase assays indicated that PAIPP2 could also interact with AIPP3 and CPL2, but not with AIPP2 (Fig. $1 \mathrm{~b}$ and Supplementary Fig. 2). Regarding the domain requirements for protein interactions, the $\mathrm{Y} 2 \mathrm{H}$ results indicated that the $\mathrm{BAH}$ domain-containing $\mathrm{N}$-terminus of AIPP3 and the RBM motif-containing C-terminus region of CPL2 are required for their interactions with AIPP2 and PAIPP2, respectively (Supplementary Fig. 3). AIPP2 and PAIPP2 were divided into three parts: the N-terminus (N), PHD that is followed by a frequently associated polybasic region (PHD-PBR), and the C-terminus (C) (Supplementary Fig. 4). The PHD-PBR part is indispensable for AIPP2/PAIPP2-AIPP3 interactions. Intriguingly, the PHD-PBR interaction with AIPP3 could be strengthened, and inhibited by the $\mathrm{N}$ and $\mathrm{C}$ termini of AIPP2/ PAIPP2, respectively (Supplementary Fig. 4), indicating the presence of an intramolecular regulation mode within AIPP2 and PAIPP2. The C termini of AIPP2 and PAIPP2 were fully responsible for the interaction with CPL2. Thus, we reasoned that the PHD proteins AIPP2 and PAIPP2 interact with AIPP3 and CPL2 independently to associate in vivo (Fig. 1c). Moreover, the gel filtration assay that was performed using epitope-tagged transgenic lines indicated that these four proteins co-eluted in the same fractions (Fig. 1d). Thus, these data support the notion that the BAH protein AIPP3 associates with two PHD proteins and CPL2 to form a protein complex in vivo (which are hereafter referred to as the BAH-PHD-CPL2 complex or the BPC complex).

The BPC complex represses flowering by inhibiting $F T$ expression. By generating native promoter-driven $\beta$ glucuronidase (GUS) reporter transgenes, we observed that the BPC complex genes were ubiquitously expressed in Arabidopsis (Supplementary Fig. 5). To explore the biological function of the BPC complex, the null mutants of PAIPP2 were generated using CRISPR/Cas9-mediated gene editing (Supplementary Fig. 6), and the morphology and flowering phenotypes of the generated paipp2-1 mutant, as well as the reported aipp3-1, aipp2-1, and cpl2-2 mutants were investigated ${ }^{41}$. The aipp3-1, cpl2-2, and aipp2-1/paipp2-1 displayed multiple developmental defects, such as a dwarfed size, small leaves, and poor fertility (Fig. 2a). Instead, the aipp2-1 and paipp2-1 mutants only showed mild 
a

\begin{tabular}{|} 
Immunoprecipitation-mass spectrometry \\
\begin{tabular}{|c|c|c|c|c|}
\hline $\begin{array}{c}\text { Baits } \\
\text { Potid }_{e_{S}}\end{array}$ & AIPP2-4Myc & AIPP3-3Flag & CPL2-4Myc & PAIPP2-3Flag \\
\hline Proteins & 78 & 9 & 9 & $/$ \\
\hline AIPP2 & 34 & 128 & 1 & 40 \\
\hline AIPP3 & 22 & 4 & 138 & 85 \\
\hline CPL2 & $/$ & 22 & 40 & 169 \\
\hline PAIPP2 & & & & \\
\hline
\end{tabular}
\end{tabular}

c

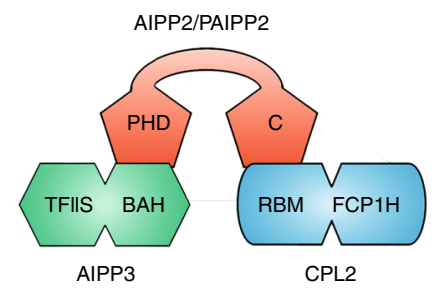

b

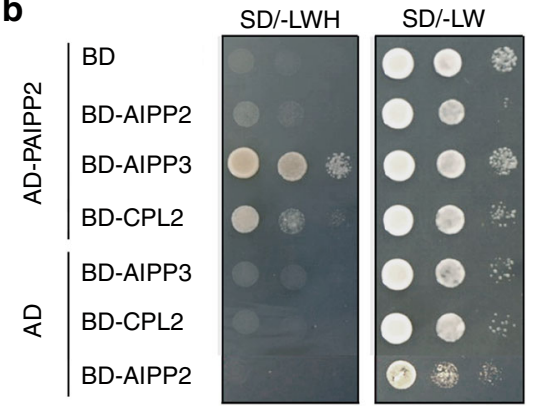

d

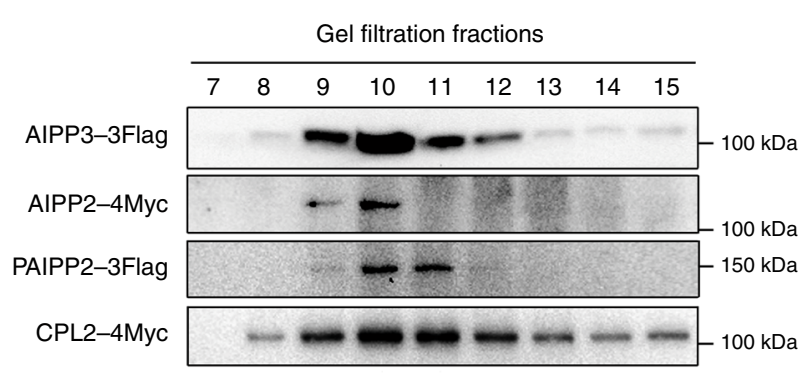

Fig. 1 The BAH protein AIPP3 associates with PHD proteins and CPL2 to form a protein complex. a Mass spectrometry analysis of epitope-tagged AIPP3, AIPP2, PAIPP2, and CPL2 transgenic plants. b $\mathrm{Y} 2 \mathrm{H}$ results showing the reciprocal interactions within the tested proteins. $\mathbf{c} A$ diagram showing the interaction network within AIPP3, AIPP2, PAIPP2, and CPL2. d Immunoblotting results of gel filtration assays. One of three independent experiments was shown.

developmental defects. Moreover, the aipp3-1 and cpl2-2 mutants showed obvious earlier flowering during both long day (LD) and short day (SD) photoperiods in comparison with Col-0 (Fig. 2b, c). Although only mild early flowering was observed in the aipp21 and paipp2-1 single mutants, aipp2-1/paipp2-1showed similar early flowering compared with the aipp3-1 and cpl2-2 mutants (Fig. 2b, c), suggesting a redundancy in these two PHD proteins in relation to the flowering time control. To dissect their genetic relationship, we attempted to generate double, triple, and quadruple mutants. Unfortunately, we failed to obtain the aipp3/aipp2/ paipp2/cpl2 quadruple mutant due to a severe developmental defect. Compared to Col-0, the aipp3-1/aipp2-1/paipp2-1, aipp3$1 / c p l 2-2$, and aipp2-1/paipp2-1/cpl2-2 mutants flowered earlier, and the time to flower was similar to the single mutants under the LD condition (Fig. 2d), suggesting that the BPC complex acts in the same genetic pathway in flowering time control.

In Arabidopsis, FLC, which is a MADS-box transcription factor that integrates multiple flowering signals, acts as a key floral repressor $^{31,43,44}$. FLC directly represses the expression of florigen gene FT and SUPPRESSOR OF OVEREXPRESSION OF CO 1 (SOC1) by binding to the promoter of SOC1 and the first intron of $F T^{45}$. The quantitative RT-PCR (RT-qPCR) indicated that the FLC RNA levels were reduced in the aipp3-1, aipp2-1, cpl2-2, paipp2-1, and aipp2-1/paipp2-1 mutants compared to Col-0 (Fig. 2e). Instead, the FT RNA levels were significantly increased in the aipp3-1, cpl2-2, and aipp2-1/paipp2-1 mutants, but not in the aipp2-1 and paipp2-1 single mutants, further supporting the functional redundancy of AIPP2 and PAIPP2. By contrast, the SOC1 RNA level was not significantly changed in all the tested mutants. It is known that the accumulation of FT protein has a circadian rhythm that peaks before dusk during LD photoper$\operatorname{iod}^{46-48}$. We noticed that the loss of the BPC complex did not change the circadian rhythm of FT mRNA, but led to a constitutive increase (Fig. 2f). To determine the genetic relationship between FLC, FT, and the BPC complex in relation to the flowering time control, $f l c-3$, and $f t-10$ mutants (in Col-0 background) were crossed with the tested mutants. Surprisingly, the aipp3-1/flc-3, cpl2-2/flc-3, and aipp2-1/paipp2-1/flc-3 mutants displayed similar early flowering compared with the aipp3-1, cpl2-2, and aipp2-1/paipp2-1 mutants, but they flowered earlier than the $f l c-3$ single mutant (Fig. $2 \mathrm{~g}$ ). By contrast, the early flowering phenotypes of aipp3-1, cpl2-2, and aipp2-1/paipp2-1 mutants were completely rescued by $f t-10$ (Fig. $2 \mathrm{~h}$ ), indicating that the BPC complex represses flowering primarily by repressing the expression of FT.

The AIPP3-BAH domain specifically recognizes the H3K27me3 mark. The chromatin-based mechanisms play vital roles in the flowering time control ${ }^{1,49,50}$. The $\mathrm{BAH}$ domain is commonly identified as an epigenetic reader module of a particular histone mark ${ }^{18}$. To decipher the molecular function of the AIPP3-BAH domain (Fig. 3a), we firstly performed a histone peptide pull-down assay using purified AIPP3-BAH protein. Among all the tested histone peptides, AIPP3-BAH could only be pulled down by $\mathrm{H} 3 \mathrm{~K} 27 \mathrm{me} 1, \mathrm{H} 3 \mathrm{~K} 27 \mathrm{me} 2$, and H3K27me3 peptides in a sequentially increasing manner (Fig. 3b). The H3K27me3-binding activity was further confirmed by isothermal titration calorimetry (ITC) binding analysis (Fig. 3c). Among all four tested histone methylation marks, H3K4me3, H3K9me2, $\mathrm{H} 3 \mathrm{~K} 27 \mathrm{me} 3$, and H3K36me3, the AIPP3-BAH domain showed a significant preference for the H3K27me3 mark. Moreover, consistent with the histone pull-down result, AIPP3-BAH has a preference for the higher methylation level in H3K27 (Fig. 3c). Thus, the evidence above fully demonstrated that AIPP3-BAH is a H3K27me3-reader module.

Structure of the AIPP3-BAH domain in complex with an H3K27me3 peptide. To gain molecular insight into the interaction between the AIPP3-BAH domain and H3K27me3, we successfully determined the crystal structure of the AIPP3-BAH domain in complex with an $\mathrm{H} 3 \mathrm{~K} 27 \mathrm{me} 3$ peptide at a resolution of $2.4 \AA$ (Fig. 3d and Supplementary Table 1). Overall, the AIPP3BAH domain has a classic $\beta$-barrel structure that is similar to other reported BAH domain structures ${ }^{18}$. The H3K27me3 peptide has a good electron density map and a $\beta$-strand-like extended conformation (Supplementary Fig. 7a). The peptide is captured by a negatively charged cavity that is formed on the surface of the 
AIPP3-BAH domain with extensive hydrophobic and hydrophilic interactions (Fig. 3e). In detail, the three aromatic residues of the AIPP3-BAH domain, Tyr149, Trp170, and Tyr172, form an aromatic cage to accommodate the trimethyl-lysine of the H3K27me3 peptide (Fig. 3f), and it resembles other typical methyl-lysine-reading histone readers ${ }^{51}$. At the N-terminus, the H3A25 forms two main chain-main chain hydrogen bonds with the AIPP3 Val140 and Glu142 (Fig. 3f). In the middle, the AIPP3 residues Trp170, Tyr172, His198, and Asp200 form an extensive hydrogen-binding network with H3S28, which further fixes the imidazole ring of His198 in a special rotamer state that is parallel with the prolyl ring of $\mathrm{H} 3 \mathrm{P} 30$. This parallel alignment of the two rings enables the $\mathrm{CH}-\pi$, and stacking interactions between AIPP3 His198 and H3P30, which resemble the recognition of

a
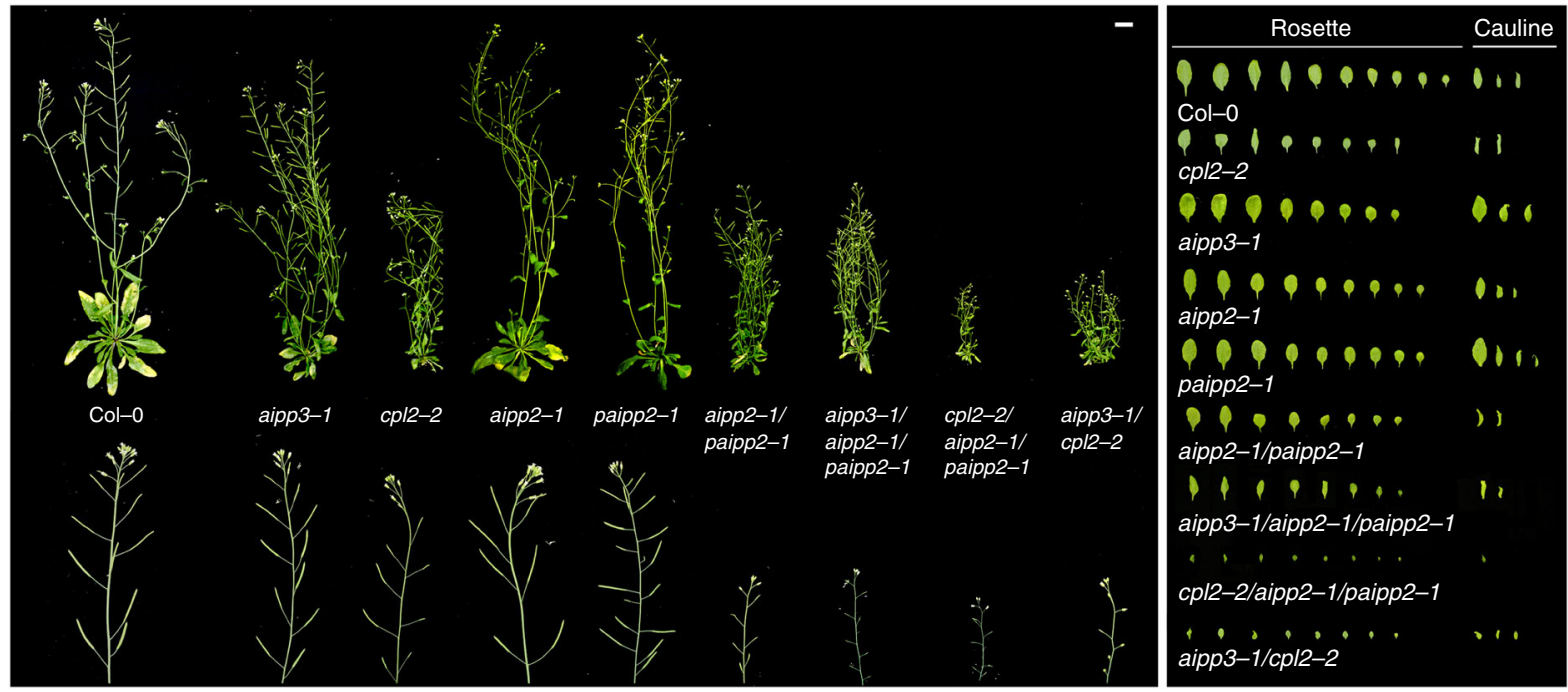

b

Col-0 aipp3-1 cpl2-2 aipp2-1 paipp2-1 paipp2-1
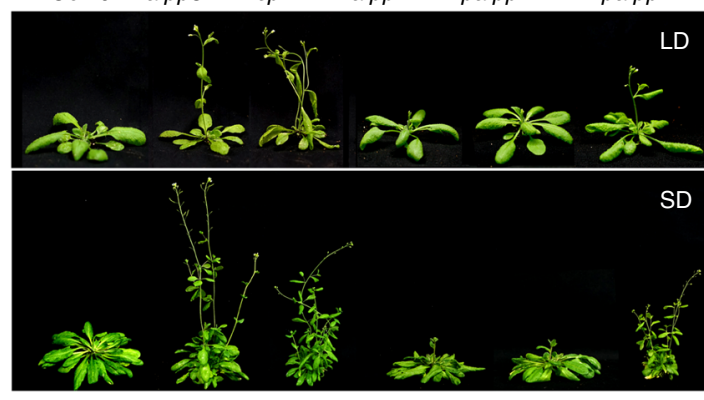

C

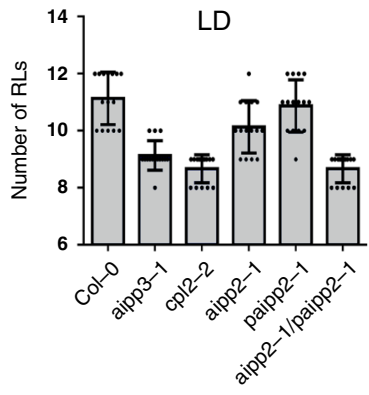

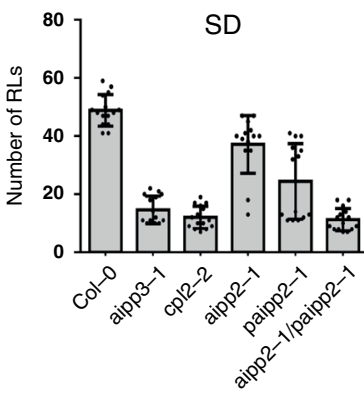

d

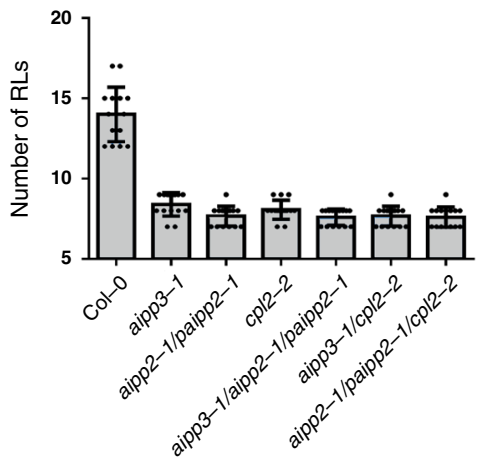

$\mathbf{f}$

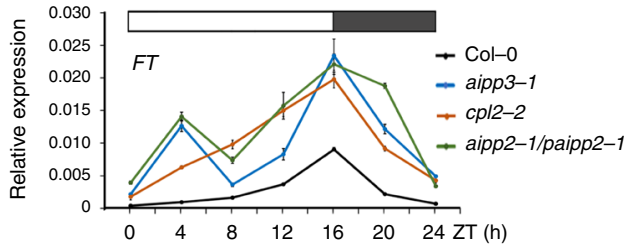

e
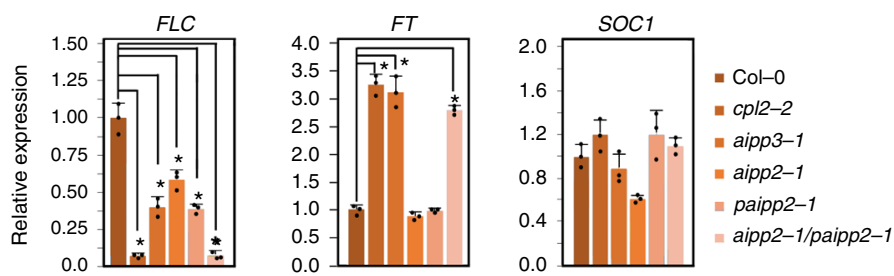

g

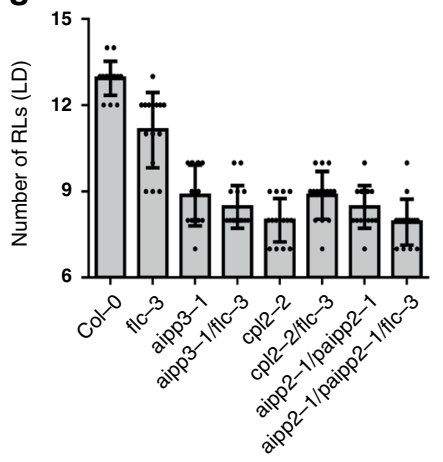

h

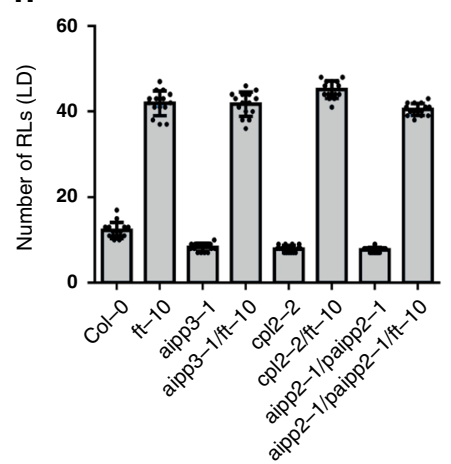


Fig. 2 The BAH-PHD-CPL2 complex regulates plant development and flowering time. a The phenotypic developmental defects of $b p c$ mutants. The morphological phenotypes of whole plants, inflorescence tissues, rosette, and cauline leaves were shown. b, c The flowering phenotypes (b) and the numbers of rosette leave (RLs) at flowering (c) in selected mutants during LD and SD photoperiods. Black horizontal lines represent the mean, and the error bars represent \pm S.D. from the number of plants counted for each genotype. Col-0: $n=15$ (LD/SD), aipp3-1: $n=15$ (LD/SD), cp/2-2: $n=15$ (LD/SD), aipp2-1: $n=15 / 14$ (LD/SD), paipp2-1: $n=15 / 14$ (LD/SD), aipp2-1/paipp2-1: $n=15$ (LD/SD). d Comparison of the number of RLs at flowering in selected mutants under the LD condition. Black horizontal lines represent the mean, and the error bars represent $\pm S$.D. from the number of plants counted for each genotype. $n=15$ per line. e The relative mRNA levels of the FLC, FT, and SOC1 genes in the selected mutants. The mRNA levels were first normalized to $A C T 2$ and then to Col-0. The data are the means \pm S.D. of three biological repeats. Unpaired one-tailed $t$ test was performed and ${ }^{\star} p$ value $<0.01$. $\mathbf{f}$ The circadian accumulation of $F T$ mRNA in selected mutants. The $F T$ mRNA levels were normalized to ACT2. The data are the means \pm S.D. of three biological repeats. The white and black boxes represent light and dark periods, respectively. $\mathbf{g}$, $\mathbf{h}$ The numbers of RLs in the BAH-PHD-CPL2 complex mutants and their double mutants with $\mathrm{flc}-3(\mathbf{g})$ and $\mathrm{ft}-10(\mathbf{h})$ at flowering during the LD photoperiod. Black horizontal lines represent the mean, and the error bars represent \pm S.D. from the number of plants counted for each genotype. $n=15$ per line.

H3K27me3 by the EBS and SHL BAH domains with conserved key residues (Supplementary Fig. $7 \mathrm{~b})^{27,28}$. In the C-terminus, H3G33 interacts with AIPP3 Val240 through a main chain-main chain hydrogen bond. To validate these structural observations, we performed a mutagenesis analysis on the key residues. The mutations D200A and H198A, which are essential for H3P30 recognition, in addition to Y149A, W170A, and Y172A, which represent important aromatic cage residues, showed reduced or no detectable binding to the H3K27me3 peptide (Fig. 3g, h).

PHD fingers of AIPP2 and PAIPP2 recognize the unmodified H3K4 mark. In addition to AIPP3-BAH, the PHD fingers of AIPP2 and PAIPP2 may also be involved in the recognition of histone marks. AIPP2 and PAIPP2 share a conserved PHD finger in their sequences (Supplementary Fig. 8a), and their sequences of $\mathrm{PHD}$ fingers possess the typical signatures of unmodified $\mathrm{H} 3$ recognition PHD fingers ${ }^{19}$. We first detected their histone substrate binding properties by ITC method. Although the AIPP2PHD finger does not behave well in vitro and tends to precipitate, we successfully detected the binding between the PAIPP2-PHD finger and the differentially methylated $\mathrm{H} 3 \mathrm{~K} 4$ peptides (Fig. 3i). The PAIPP2-PHD finger prefers to bind to unmethylated H3K4 and the binding affinities were clearly decreased when the methylation level of the $\mathrm{H} 3 \mathrm{~K} 4$ peptide was increased. Considering the high sequence similarity between the two PHD fingers (Supplementary Fig. 8a), we believe that the AIPP2-PHD finger may possess the same binding preference on the unmodified H3K4. Moreover, both AIPP2 and PAIPP2-PHD fingers share $\sim 35 \%$ sequence identity with the PHD finger of Glycine max ATXR5 (PDB code: 5VAB; Supplementary Fig. 8a), which recognizes the unmodified $\mathrm{H} 3 \mathrm{~K} 4$ (ref. ${ }^{52}$ ). We modeled the AIPP2 and PAIPP2-PHD fingers using the ATXR5 PHD finger, as a template to analyze the interactions with unmodified $\mathrm{H} 3 \mathrm{~K} 4$ (Fig. $3 \mathrm{j}$ and Supplementary Fig. 8b). In the modeled structure, we noticed that almost all the peptide-binding residues are conserved (Supplementary Fig. 8a). For instance, the Pro322 and Gly324 of PAIPP2, which correspond to the ATXR5 Pro60 and Gly62, respectively, are involved in the hydrogen-binding interaction with H3A1 (Fig. 3k). Similarly, the Ile300, Cys301, and Asp306 of PAIPP2, which are equivalent to ATXR5 Leu39, Cys40, and Asp44, respectively, contribute to the recognition of H3R2 (Fig. 3k). The Val285, Gly292, and Leu298 of PAIPP2, which correspond to the Val23, Gly31, and Leu37 of ATXR5, respectively, participate in the recognition of the unmodified H3K4 (Fig. 3k). To validate the modeling results, we performed mutagenesis experiment, too. As most of the peptide recognition is achieved by the hydrogen-bonding interactions of the main chain of the PHD finger, we only mutated Asp306 of PAIPP2, which is involved in the recognition of H3R2 by its side chain. As shown in Fig. 31, the D306K mutation of PAIPP2-PHD finger almost totally disrupts the peptide binding, further supporting our modeling data. The AIPP2-PHD finger possesses similar unmodified $\mathrm{H} 3 \mathrm{~K} 4$ recognition residues and interactions (Supplementary Fig. 8c).

The BPC complex represses the expression of the genes marked by $\mathrm{H} 3 \mathrm{~K} 27 \mathrm{me} 3$ and low-methylated H3K4. H3K27me3 is usually considered a repressive mark that functions in transcriptional repression by recruiting recognition proteins or protein complexes ${ }^{4,8}$. To dissect the molecular function of the BPC complex, mRNA-seq was performed using their null mutants, as well as CLF and LHP1 mutants ${ }^{53,54}$, which are one of the known $\mathrm{H} 3 \mathrm{~K} 27 \mathrm{me} 3$ methyltransferases and a reader protein in Arabidopsis, respectively. Using a twofold change cutoff, we noticed that the numbers of upregulated differentially expressed genes (up-DEGs) were far greater than the numbers of down-DEGs in the aipp3-1, cpl2-2, and aipp2-1/paipp2-1 mutants (Fig. 4a). Supporting the functional redundancy of AIPP2 and PAIPP2, very few DEGs were identified in aipp2-1 and paipp2-1 single mutants. It is noteworthy that most of the up-DEG genes regulated by the BPC complex display very low-expression levels in the wild type, which is a similar pattern to that the clf and $l h p 1$ mutants (Fig. 4b). Under a strict criterion, 155 genes were commonly upregulated in the bah-phd-cpl2 mutants (Figs. $4 \mathrm{c}$, $\mathrm{d}$ and Supplementary Data 2), and a substantial subset are stressresponsive genes (Supplementary Fig. 9), such as SEC31A (AT1G18830), which participates in endoplasmic reticulum stress responses ${ }^{55-57}$, and AGO5 (AT2G27880), which is involved in antiviral RNA silencing ${ }^{58-60}$ and gametophyte development ${ }^{61,62}$. In fact, for most of the aipp2-1/paipp2-1 up-DEGs, higher expression was also observed in the aipp3-1 and cpl2-2 mutants (Fig. 4d), indicating that the BPC complex targets a common subset of genes for repression.

We next explored the chromatin feature of the target genes by plotting the distributions of the histone marks on the up-DEGs, using published histone modifications chromatin immunoprecipitation (ChIP)-seq data ${ }^{63}$. As expected, the H3K27me3 mark was heavily enriched on the bodies of the common target genes (Fig. 4e). Consistent with the ITC result in which the higher methylation of $\mathrm{H} 3 \mathrm{~K} 4$ inhibits the binding of PAIPP2-PHD (Fig. 3i), the levels of active H3K4me3 mark were lower in the regions around the TSS. H3K36me3 deposition was also low on the whole gene bodies. The depositions of $\mathrm{H} 3 \mathrm{~K} 9 \mathrm{me} 2$ and H3K27me1 were markedly lower on the target genes, suggesting that the BPC complex primarily targets euchromatic genes. The distribution patterns of $\mathrm{H} 3 \mathrm{~K} 27 \mathrm{me} 3$ and $\mathrm{H} 3 \mathrm{~K} 4 \mathrm{me} 3$ marks at BPC complex target genes were confirmed by ChIP-qPCR assays at representative target genes $A G O 5, S U C 5$, and FT (Fig. 4f, g). We next investigated the impacts of BPC complex dysfunction on global histone mark levels. Interestingly, compared to the reduction of global $\mathrm{H} 3 \mathrm{~K} 27 \mathrm{me} 2 / 3$ levels in clf-81 mutant, the absence of the BPC complex did not result in obvious changes in 
global levels of H3K27me1/2/3 and H3K4me1/2/3 (Supplementary Fig. 10a). Consistent with this pattern, H3K27me3 deposition was not obviously changed between Col-0 and $b p c$ mutants at selected target genes AGO5, SUC5, and FT (Fig. 4g), whereas $\mathrm{H} 3 \mathrm{~K} 4 \mathrm{me} 3$ levels are slightly increased (Fig. 4h). To further verify this observation, more target genes were selected. As shown in Supplementary Fig. 10b-d, nuclear run-on assay showed that AT2G43570, AT3G59480, and AT3G53650 genes were transcriptionally upregulated compared to wild type, indicating that these three genes are subjected to BPC complex-mediated transcriptional repression. Interestingly, ChIP-qPCR results indicated that $\mathrm{H} 3 \mathrm{~K} 27 \mathrm{me} 3$ levels were not obviously changed in bpc mutants at AT2G43570 and AT3G59480, consistent with our observation at AGO5, SUC5, and FT (Fig. 4g). While H3K27me3 deposition showed significant reduction at AT3G53650, implying that BPC dysfunction has substantial impacts on H3K27me3 deposition at this gene. Combined with these data, we speculate that, for most target genes, the BPC complex may serve as a surveillance system to prevent reactivation of $\mathrm{H} 3 \mathrm{~K} 27 \mathrm{me} 3$-marked genes, which are already silenced by PRC2, but in some specific a
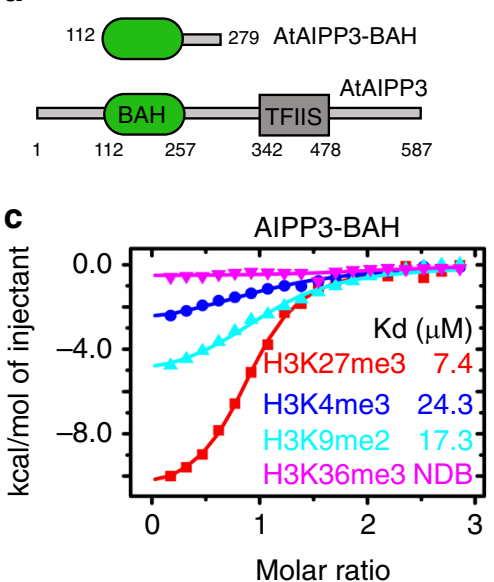

e

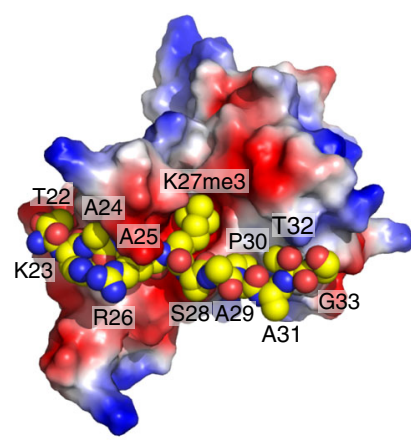

$\mathbf{g}$

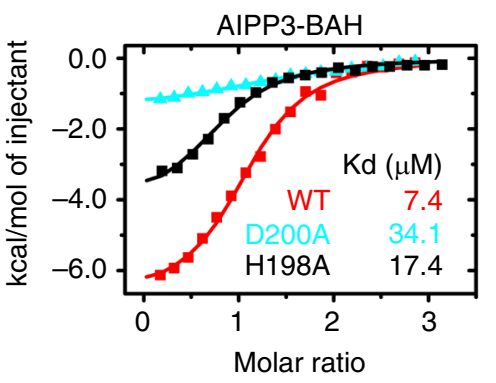

j

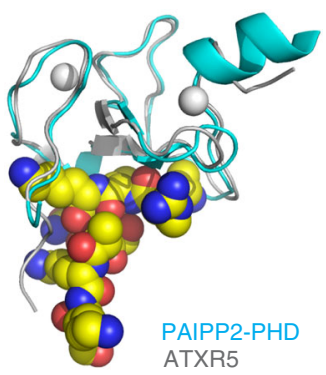

b
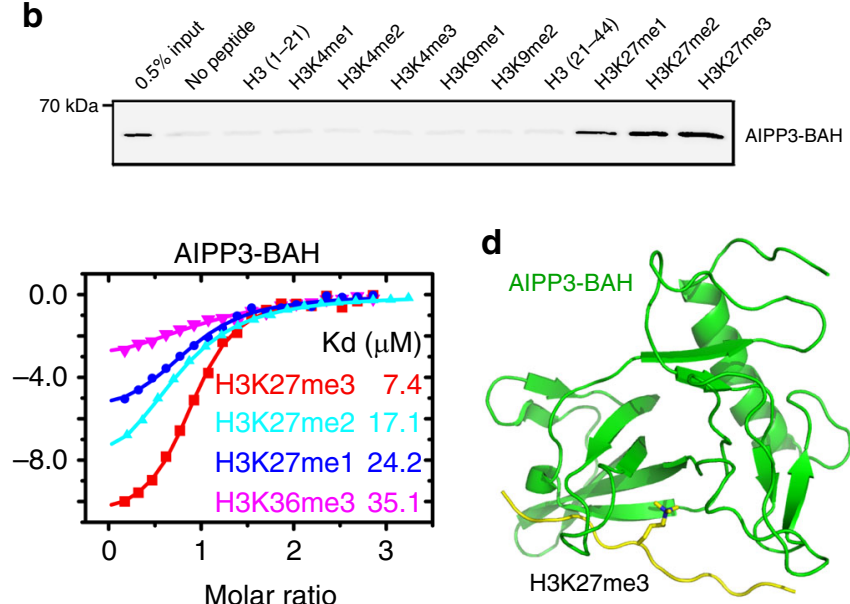

$\mathbf{f}$

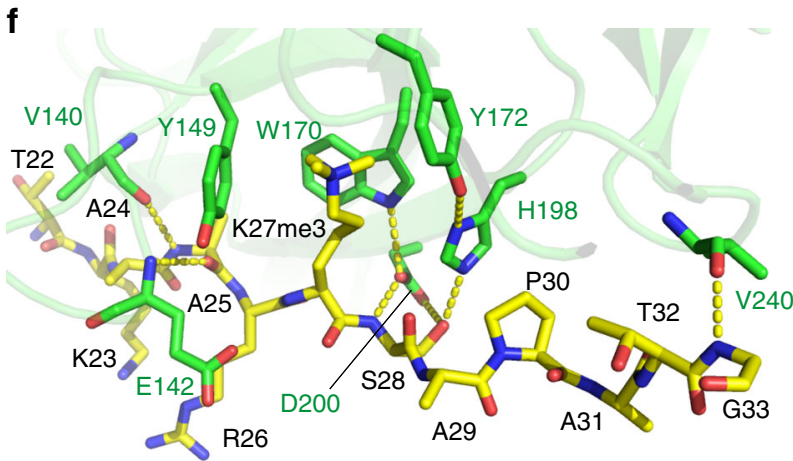

h

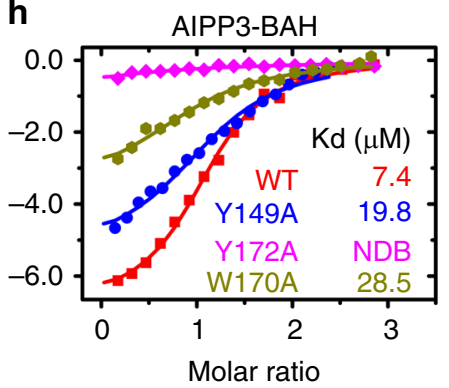

i

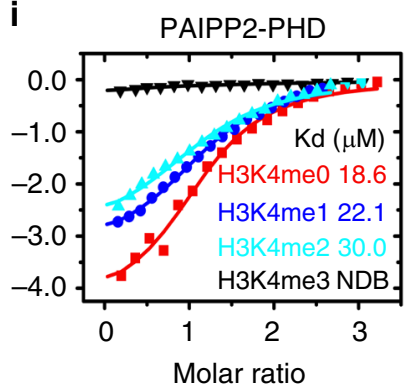

$\mathbf{k}$

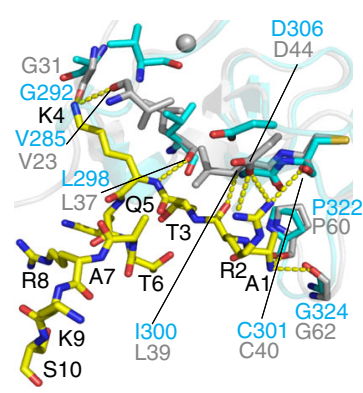

I

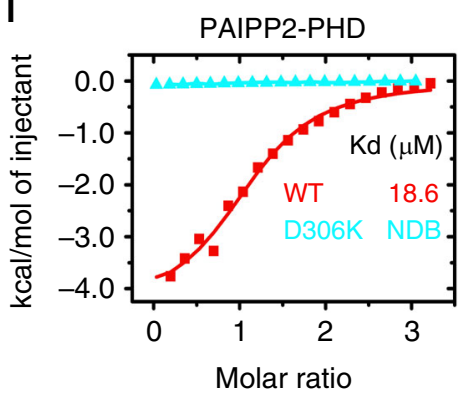


Fig. 3 Structural analysis of the AIPP3-BAH domain and PAIPP2-PHD finger in complex with H3K27me3 and unmodified H3K4 peptide, respectively. a The domain architecture of AIPP3 (lower panel) and the BAH domain construct used for structural and biochemical studies (upper panel). b The immunoblotting results showing the pull-down results for AIPP3-BAH using different histone peptides. The $0.5 \%$ inputs serve as positive controls. c The ITC binding curves showing the AIPP3-BAH domain-binding preference for different histone methylation marks (left panel) and different H3K27 methylation levels (right panel). NDB no detectable binding. d The overall structure of the AIPP3-BAH domain H3K27me3 peptide complex shown in ribbon with the AIPP3-BAH domain, and the H3K27me3 peptide colored in green and yellow, respectively. e An electrostatic surface view of AIPP3-BAH domain with the H3K27me3 peptide in the space-filling model showing that the peptide fits inside a negatively charged surface cleft of the AIPP3-BAH domain. $\mathbf{f}$ The detailed interaction between AIPP3-BAH domain and the H3K27me3 peptide with the interacting residues highlighted in sticks and the hydrogen bonds highlighted in dashed yellow lines. $\mathbf{g}$, $\mathbf{h}$ The ITC binding curves show that the mutations of essential residues for the H3P30 recognition ( $\mathbf{g}$ ) and aromatic cage residues (h) of the AIPP3-BAH domain significantly decrease the binding toward the H3K27me3 peptide. $\mathbf{i}$ The ITC binding curves showing the specific preference of the PAIPP2-PHD finger for the unmodified H3K4. $\mathbf{j}$ The overall modeled structure of the PAIPP2-PHD finger in complex with the unmodified H3 peptide with the PHD finger and peptide shown in ribbon and space-filling models. The modeled PAIPP2-PHD finger and the modeling template ATXR5 PHD finger are colored in cyan and silver, respectively, and they were superimposed together. $\mathbf{k}$ The detailed interaction between the PAIPP2-PHD finger (in cyan) and the unmodified H3K4 peptide with the interacting residues are highlighted in stick model and hydrogen bonds highlighted in dashed yellow lines. The corresponding residues from modeling template ATXR5 (in silver, PDB code: 5VAB) were overlain and highlighted, showing that almost all the interacting residues are conserved. I The ITC binding curves showing that the D306K mutation of PAIPP2-PHD finger, which potentially disrupts H3R2 recognition, totally abolishes the unmodified H3K4 binding by PAIPP2.

genes, BPC is required for $\mathrm{H} 3 \mathrm{~K} 27 \mathrm{me} 3$ deposition through unknown mechanism.

The BPC complex reads the $\mathrm{H} 3 \mathrm{~K} 27 \mathrm{me} 3$ at a genome-wide level. We next investigated the interplay between the BPC complex and the chromatin of the target genes by performing a ChIP assay in epitope-tagged transgenic plants. The ChIP-qPCR results indicated that AIPP3, AIPP2, and PAIPP3 were enriched in the selected target genes, particularly in the regions close to TSSs (Fig. 5a), but they were low in the adjacent high H3K4me3/low H3K27me3 genes. To confirm this point, an AIPP3 ChIP-seq was performed. As shown in Fig. 5b, AIPP3 specifically binds to the selected target genes. At the genome-wide level, AIPP3-binding peaks were enriched in the DEGs that were upregulated in the aipp3-1 mutant (Fig. 5c, d). Moreover, a pattern of high H3K27me3 and low H3K4me3 was clearly observed in AIPP3bound genes (Fig. 5e). Next, we compared the genome-wide occupancy of AIPP3 with a published global analysis of the H3K27me3-marked regions ${ }^{1}$. As shown in Fig. 5f, 455 loci, or approximately half of the AIPP3-enriched loci, significantly overlap with $\mathrm{H} 3 \mathrm{~K} 27 \mathrm{me} 3$-enriched loci, indicating that a substantial part of the H3K27me3 loci were targeted by the AIPP3 complex. In Arabidopsis, EMBRYONIC FLOWER 1 (EMF1) is a plant-specific PRC1 component that is essential for conferring H3K27me3-dependent silencing at thousands of loci ${ }^{29}$. Recently, EMF1 has been shown to interact with two H3K27me3 readers to form the BAH-EMF1 complex and implement silencing ${ }^{29}$. We compared the AIPP3 loci with the published EMF1-bound loci and found that $~ 59 \%$ of AIPP3-enriched loci were also occupied by EMF1 (Fig. $5 \mathrm{f}$ ). While, no EMF1 peptides were found in the BPC complex co-purified proteins (Supplementary Data 1). One possible explanation is that both EMF1 and AIPP3 associate with H3K27me3 mark independently, although the possibility of indirect association between these two reader proteins cannot be excluded.

To decipher whether H3K27me3 binding is indispensable for flowering time control and transcription repression, the wild-type and mutated AIPP3 genomic DNA, in which the crucial Tyr149, Trp170, and Tyr172 residues required for H3K27me3 binding were mutated into alanine was introduced into the aipp3-1 mutant under the direction of the native promoter to generate AIPP3, W170A, and Y149A/W170A/Y172A transgenic Arabidopsis. The early flowering of the aipp3-1 mutation was rescued by the wild-type AIPP3 transgene, but not by the W170A or Y149A/ W170A/Y172A transgenes (Fig. 6a, b). Interestingly, compared to the comparable accumulation levels of AIPP3 and W170A proteins in transgenic plants, Y149A/W170A/Y172A protein level was much lower (Fig. 6c), indicating that Y149A/W170A/ Y172A mutation alters the stability of AIPP3 protein. Therefore, we used AIPP3 and W170A transgenes in the following experiment. RT-qPCR results indicated that the W170A mutation could not recover the repressive state of the selected target genes (Fig. 6d), suggesting that the H3K27me3-binding activity is essential for the repression of flowering and gene expression. To test whether W170A mutation has an impact on AIPP3 binding at target genes, ChIP-qPCR assay was performed in Col-0, AIPP3, and W170A transgenic plants. As shown in Fig. 6e, compared to the significant enrichment at selected target genes in AIPP3 transgene, AIPP3 binding was disrupted by the W170A mutation. Considering the fact that W170 is essential for H3K27me3binding activity (Fig. 3h), this result strengthens our conclusion that H3K27me3-binding activity is indispensable for AIPP3mediated flowering time control and transcriptional repression. Interestingly, the mutations did not affect the AIPP3 interactions with AIPP2 and PAIPP2 (Supplementary Fig. 11), indicating that the BPC complex is not dissociated by the disabling of H3K27me3 binding.

BAH-PHD-CPL2 couples the recognition of H3K27me3 and the repression of Pol II release. It has been well documented that differential phosphorylation on the Ser2 (Ser2P) and Ser5 (Ser5P) of the Pol II CTD plays essential roles in the switches between distinct transcriptional stages ${ }^{64}$. During transcription, Pol II is first assembled at the promoter region. After initiation, Pol II is phosphorylated at Ser5 and Ser2, and is then released from the proximal-promoter region to engage in productive elongation ${ }^{64,65}$. CPL2 has been shown to dephosphorylate the CTD-Ser5-PO4 of Pol II (ref. ${ }^{66}$ ). Therefore, it is reasonable to hypothesize that the $\mathrm{BAH}-$ and $\mathrm{PHD}$-mediated recognition of H3K27me3 and unmodified H3K4 directly represses transcription through the CPL2-mediated dephosphorylation of Pol II. To confirm this hypothesis, we first determined the states of different forms of Pol II in the target genes. Recently, Zhu et al. revealed Pol II dynamics with single-nucleotide resolution in Arabidopsis, using native elongating transcript sequencing (NET-seq) ${ }^{67}$. Surprisingly, only a sharp peak of unphosphorylated Pol II signals was observed at the TSS region of the selected target genes, and both the Ser5P and Ser2P signals were quite low or even undetectable throughout the promoter-proximal regions and gene bodies (Fig. 7a). By contrast, in the H3K4me3-enriched active expressing genes that were independent from the regulation of the BPC complex (Fig. 4f), the maximum signals for 
a

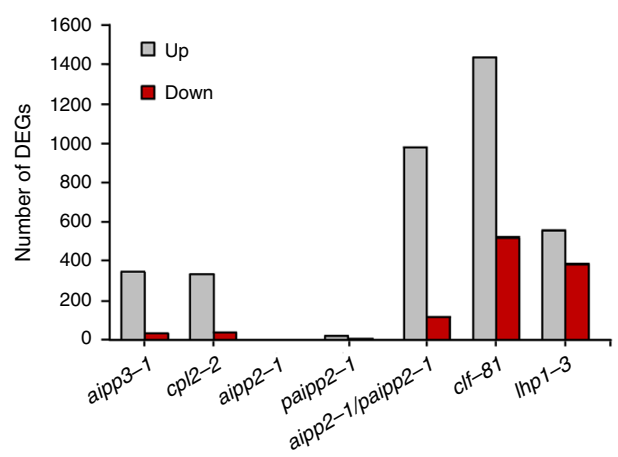

b

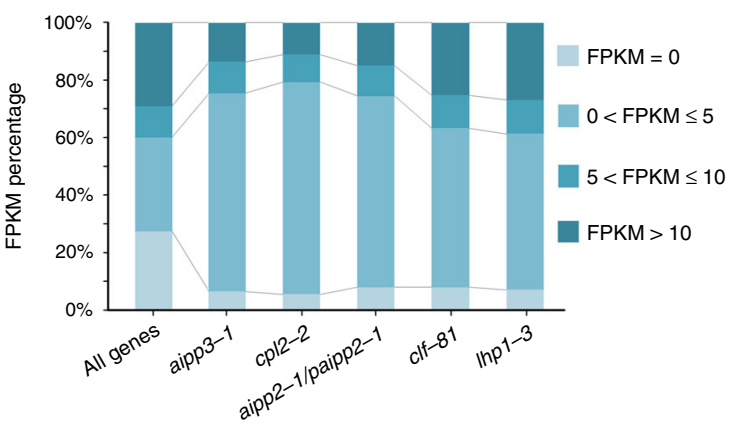

C

aipp2-1/paipp2-1 up-DEGs (977)

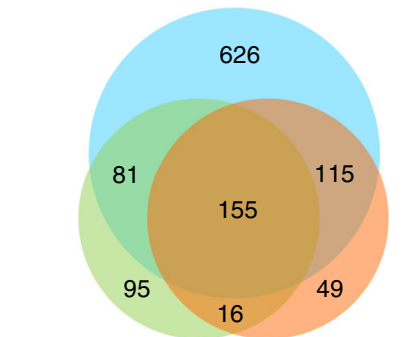

aipp3-1 up-DEGs cpl2-2 up-DEGs

(977) d

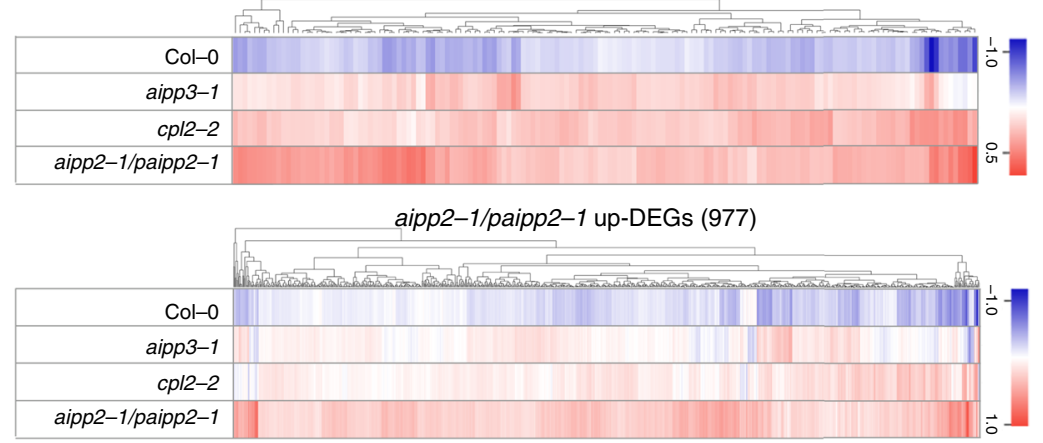

e
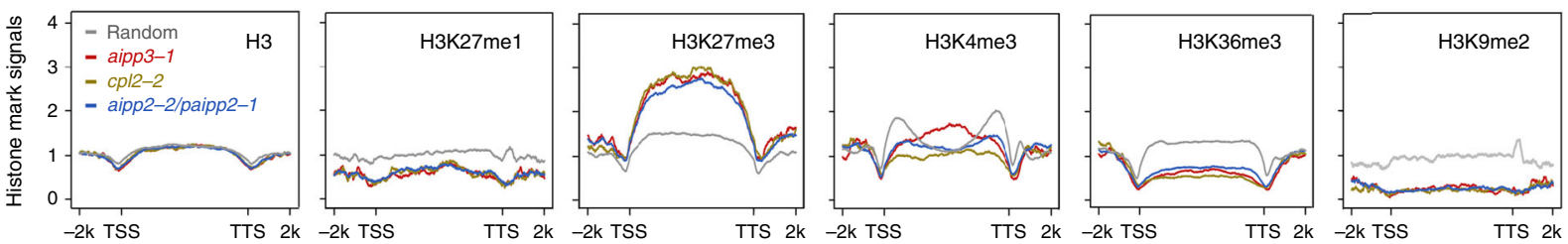

f
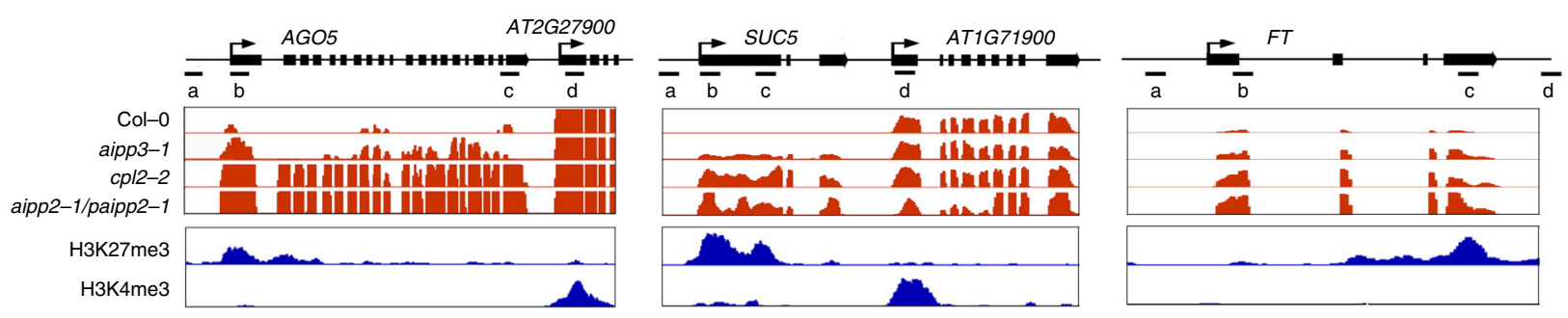

g

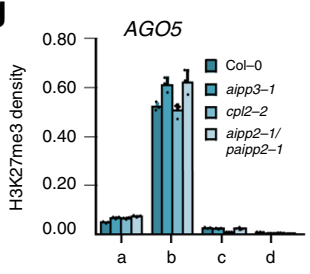

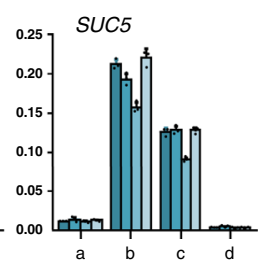

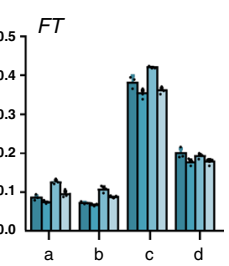

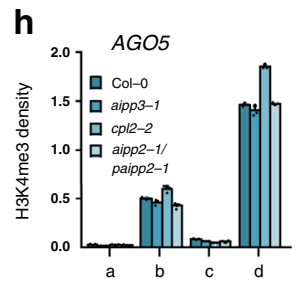

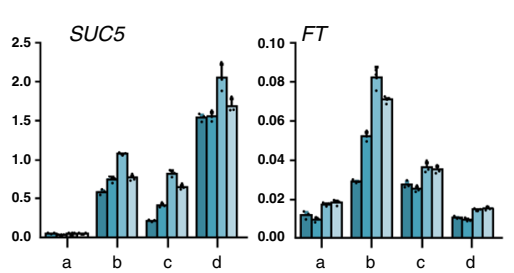

Fig. 4 BAH-PHD-CPL2 complex represses the expression of H3K27me3-enriched genes. a The numbers of up- and down-DEGs identified in the selected mutants. A twofold cutoff was used for the DEG criterion. b The BAH-PHD-CPL2 complex primarily represses low-expression genes. The gene expression levels of up-DEGs were classified into four groups according to their FPKM values in Col-0 mRNA-seq. All the annotated genes serve as controls. $\mathbf{c}$ A Venn diagram showing the overlap of up-DEGs between the selected mutants. $\mathbf{d}$ A heatmap showing the expression analysis in selected mutants. The genes in the upper and lower panels represent the common 155 up-DEGs and up-DEGs of aipp2-1paipp2-1, respectively. e The distribution of different histone marks in the respective up-DEGs of selected mutants. TTS transcription termination site. $\mathbf{f}$ Snapshots showing the expression of the selected target genes. One representative replicate of mRNA-seq and histone ChIP-seq are shown. The adjacent genes with high H3K4me3/low H3K27me3 were also shown as parallel controls. g, h ChIP-qPCR showing the H3K27me3 (g) and H3K4me3 (h) density at the selected target genes. The ChIP signals were normalized to histone H3. The data are means \pm S.D. of three technical repeats. One representative result of three biological replicates is shown. The lowercase letters represent the ChIP-qPCR examined regions as shown in $\mathbf{f}$. 

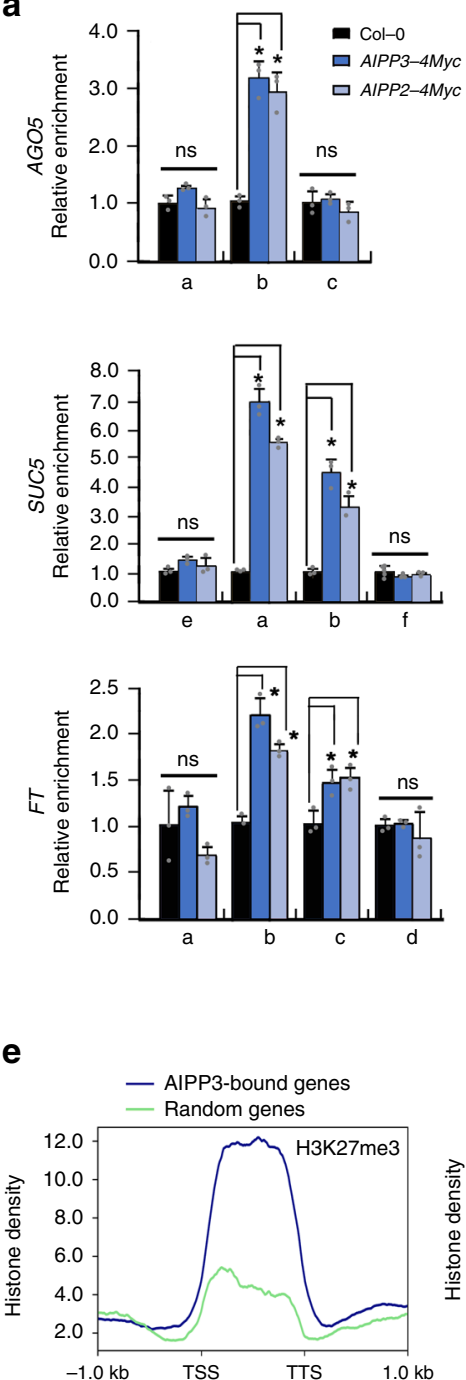
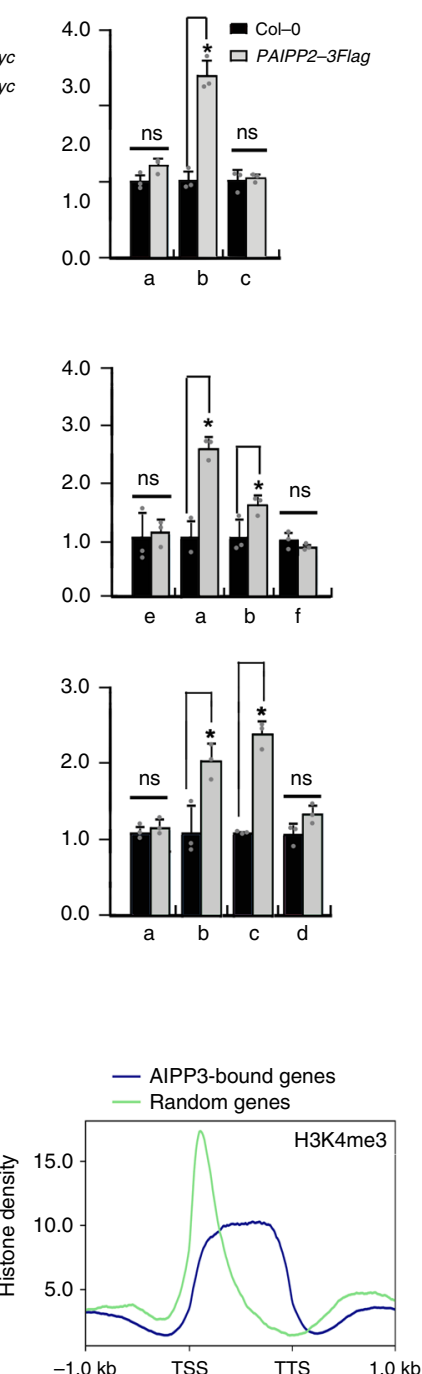

b

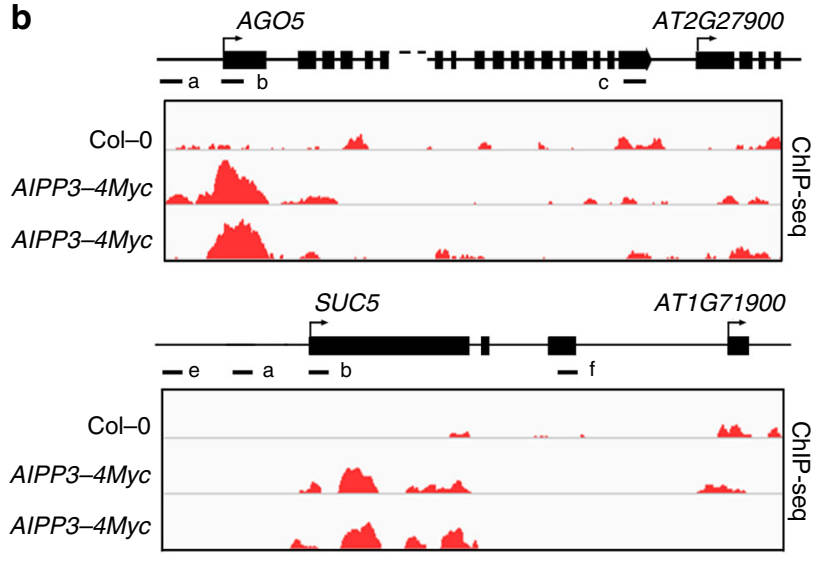

c

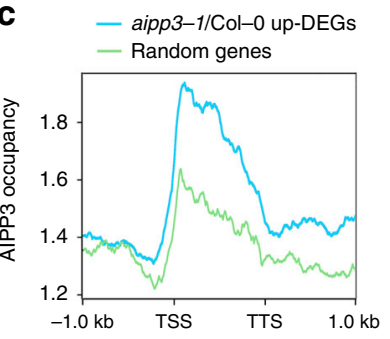

d
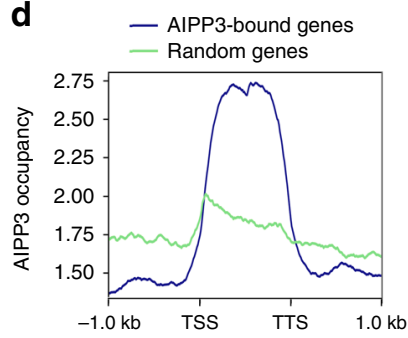

f

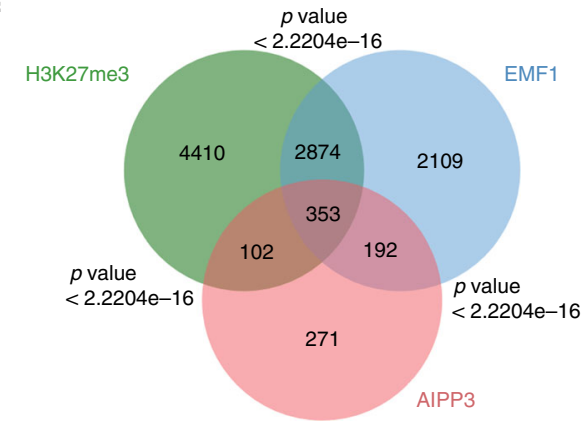

Fig. 5 BAH-PHD-CPL2 complex binds to the H3K27me3-marked genes to repress the transcriptional initiation and elongation of Pol II. a ChIP-qPCR showing the fold enrichments of AIPP3, AIPP2, and PAIPP2 on the selected genes. The occupancy was first normalized to the internal control AtSN1, and

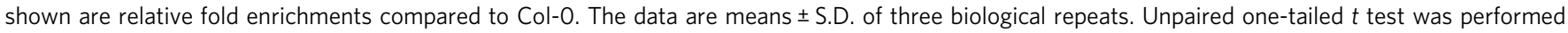
and ${ }^{*} p$ value $<0.01$. b Snapshots of AIPP3-4Myc ChIP-seq showing the distribution of the AIPP3 protein at the selected genes. Note: the ChIP-qPCR and ChIP-seq results of AIPP3-4Myc were from independent samples. c, d The diagrams showing AIPP3 occupancies on aipp3-1 up-DEGs (b) and AIPP3-bound genes (c). e The diagrams showing the H3K27me3 (left) and H3K4me3 (right) density on AIPP3-bound genes. Random regions serve as negative controls. f A Venn diagram showing the overlap between AIPP3-bound genes, H3K27me3-enriched genes, and EMF1-bound genes.

unphosphorylated Pol II were detected at approximately $+200 \mathrm{bp}$ and sharply decreased to a very low level, and Ser5P and Ser2PPol II peaked in the promoter-proximal regions, but decreased to a mild level when entering the gene body regions (Fig. 7a). These results strongly support the idea that transcription initiation and subsequent elongation of target genes were repressed in the wild type by the BPC complex. We then checked what happens to the Pol II occupancy when the complex is absent. Compared to the wild type, higher accumulations of Ser5P-Pol II were observed at the selected target genes in aipp3-1, cpl2-2, and aipp2-1/paipp2-1 mutants (Fig. 7b), and the Pol II signals maintained high levels toward the $3^{\prime}$ end of the selected genes. Similar to Ser5P-Pol II, the occupancy of total (unphosphorylated) and Ser2P-Pol II also displayed higher levels at selected target genes AGO5, SUC5, and $F T$ in the $b p c$ mutants in comparison with Col-0 (Supplementary Fig. 12). In contrast, the occupancies of all types of Pol II at AGO5 and SUC5 downstream nontarget genes were not significantly changed (Supplementary Fig. 12), demonstrating that $b p c$ mutations led to reactivation of Pol II initiation specifically at BPC complex target genes and the initiated Pol II successfully switched to an elongating state in the $b p c$ mutants. These evidence, combined with the known knowledge that CPL2 is a Ser5P-Pol II phosphatase, prompts us to hypothesize that the BPC complex represses gene expression by connecting the $\mathrm{BAH}-\mathrm{PHD}$ module-mediated recognition of $\mathrm{H} 3 \mathrm{~K} 27 \mathrm{me} 3 / \mathrm{unmo}-$ dified H3K4 and the CPL2-mediated dephosphorylation of Pol II CTD-Ser5-PO4.

To support the above hypothesis, flavopiridol (FLA) treatment assay was performed which can reduce the phosphorylation of Pol II CTD by inhibiting the activity of CDK kinases ${ }^{67}$, and the nascent RNA levels of the selected target genes were measured by nuclear run-on assay. The results indicated that the nascent RNA levels of AGO5 and SUC5, but not that of the downstream nontarget genes, were dramatically increased in the $b p c$ mutants compared to Col-0 in mock condition. This evidence strongly supports our conclusion of transcriptional repression 
a

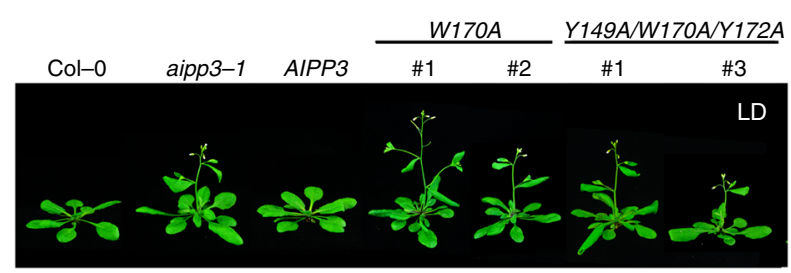

b

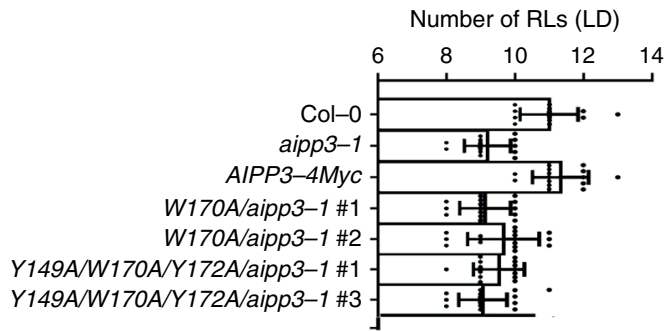

C

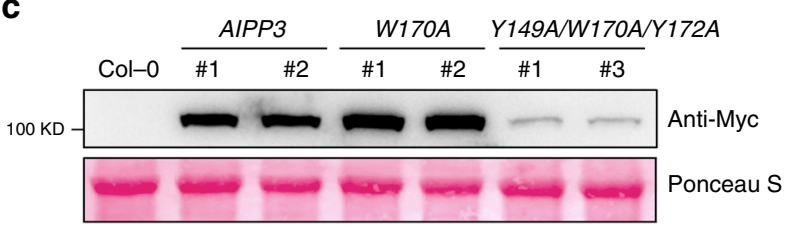

d
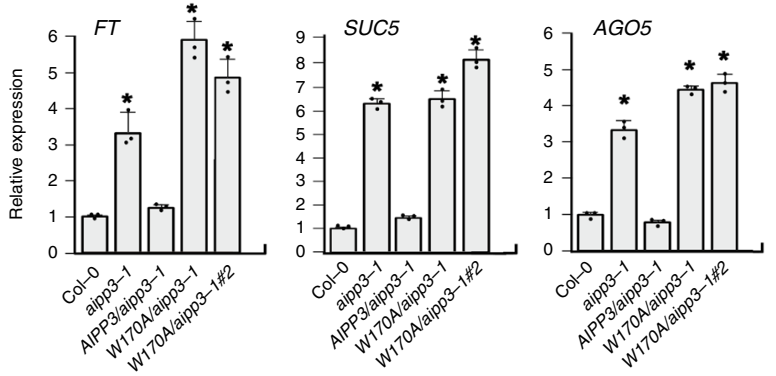

e
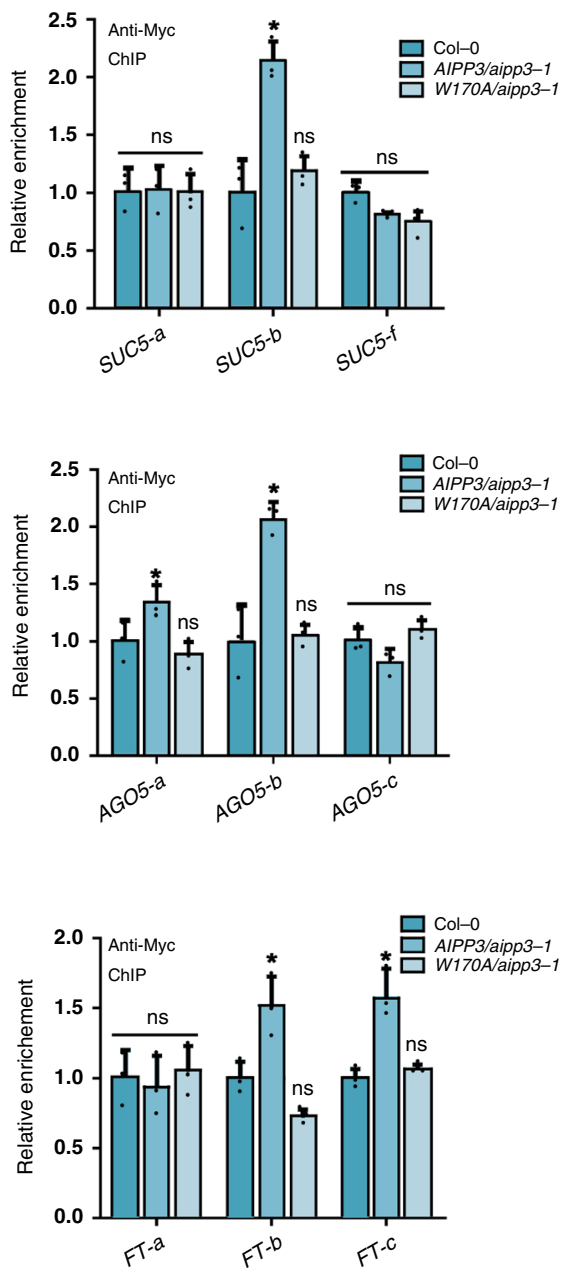

Fig. 6 The H3K27me3-binding activity is essential for AIPP3-mediated repression of flowering and gene expression. a The flowering phenotypes of the aipp3-1 mutant for its complementary lines when transformed with the wild-type AIPP3 genomic DNA, mutated AIPP3 in which the key amino acids for H3K27me3-binding were mutated. For the mutated transgene, two randomly selected transgenes were used for the analysis. The plants were grown under LD. $\mathbf{b}$ The column showing the numbers of rosette leaves (LDs) of different AIPP3 transgene plants upon flowering under the LD condition. Black horizontal lines represent the mean, and the error bars represent \pm S.D. from the number of plants counted for each genotype. $n=15$ per line. $\mathbf{c}$ Western blotting result showing the accumulation levels of AIPP3 proteins in different transgenes. Ponceaus staining serves as protein loading controls. $\mathbf{d}$ The relative mRNA levels of the selected target genes of the BAH-PHD-CPL2 complex in the wild-type, aipp3-1, and different AIPP3 transgene plants. Their levels are presented relative to Col-O. The data are the means \pm S.D. of three biological repeats. Unpaired one-tailed $t$ test was performed and ${ }^{*} p$ value $<0.01$. e ChIP-qPCR resulting showing AIPP3 occupancy at the selected target genes in AIPP3 and W170A transgenes. The data are the means \pm S.D. of three biological repeats. Unpaired one-tailed $t$ test was performed and ${ }^{*} p$ value $<0.01$. ns no significance.

implemented by the BPC complex. In contrast, the nascent RNA levels of both AGO5, SUC5, and the downstream nontarget genes were greatly reduced by FLA treatment, and no obvious changes were observed between different genotypes (Fig. 7c), indicating that inhibition of Pol II CTD phosphorylation dramatically repressed the transcription reactivation caused by the malfunctions of the BPC complex. Considering the likelihood of general effects of FLA treatment on transcriptional at a global level, to strengthen our hypothesis, the chromatin binding of CPL2 was compared in the presence or absence of AIPP3 and PHD proteins. To this end, CPL2-GFP transgene was crossed into aipp3-1 and aipp2-1/paipp2-1 mutants, and CPL2 ChIP-qPCR assay was performed in CPL2-GFP/Col-0, CPL2-GFP/aipp3-1, and CPL2-GFP/aipp2-1/paipp2-1 plants. The result indicated that CPL2 has significant binding at selected target genes in wildtype background, whereas this binding was completely abolished in the aipp3-1 and aipp2-1/paipp2-1 mutants (Fig. 7d). This result provides a link between chromatin marks and CPL2-mediated dephosphorylation of Pol II, in which the chromatin localization of CPL2 at BPC complex target genes largely depends on the recognition of $\mathrm{H} 3 \mathrm{~K} 27 \mathrm{me} 3 / \mathrm{H} 3 \mathrm{~K} 4 \mathrm{me} 0$ marks by $\mathrm{BAH}-\mathrm{PHD}$ proteins. Based on these evidences, we proposed a working model of the transcriptional repression conferred by the BPC complex (Fig. 8). In this model, the BAH-PHD bivalent histone reader recognizes $\mathrm{H} 3 \mathrm{~K} 27 \mathrm{me} 3$, and unmodified $\mathrm{H} 3 \mathrm{~K} 4$ marks and recruits CPL2 to dephosphorylate the Ser5P of Pol II CTD, resulting in the inhibition of Pol II release from a transcriptional initiation state to elongation. When the BPC complex is absent, active $\mathrm{H} 3 \mathrm{~K} 4 \mathrm{me} 3$ mark is deposited at BPC target genes. The Ser5 and Ser2 residues of Pol II CTD are sequentially phosphorylated, leading to transcriptional reactivation of BPC target genes. 
a
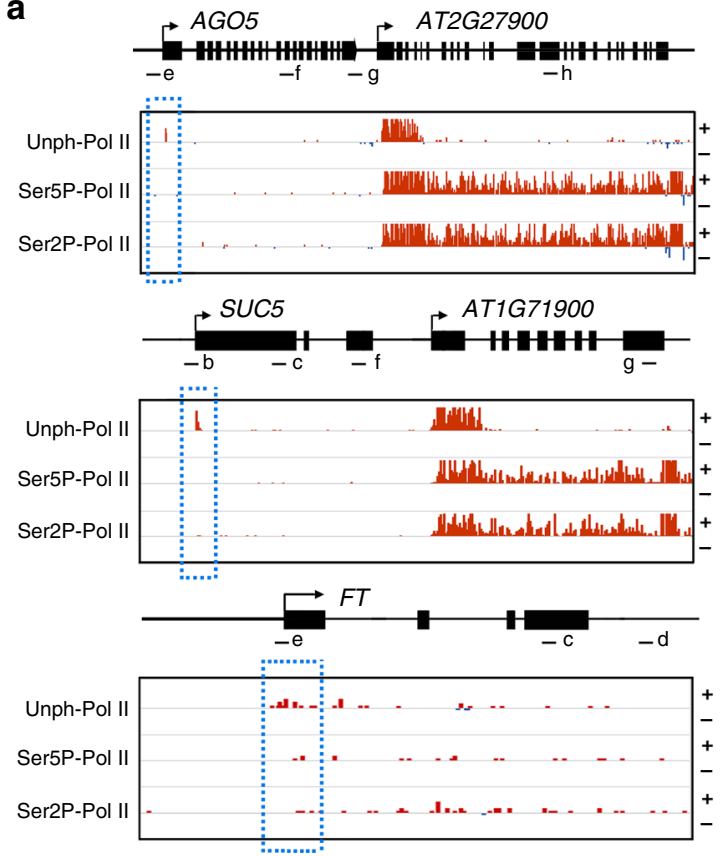

b
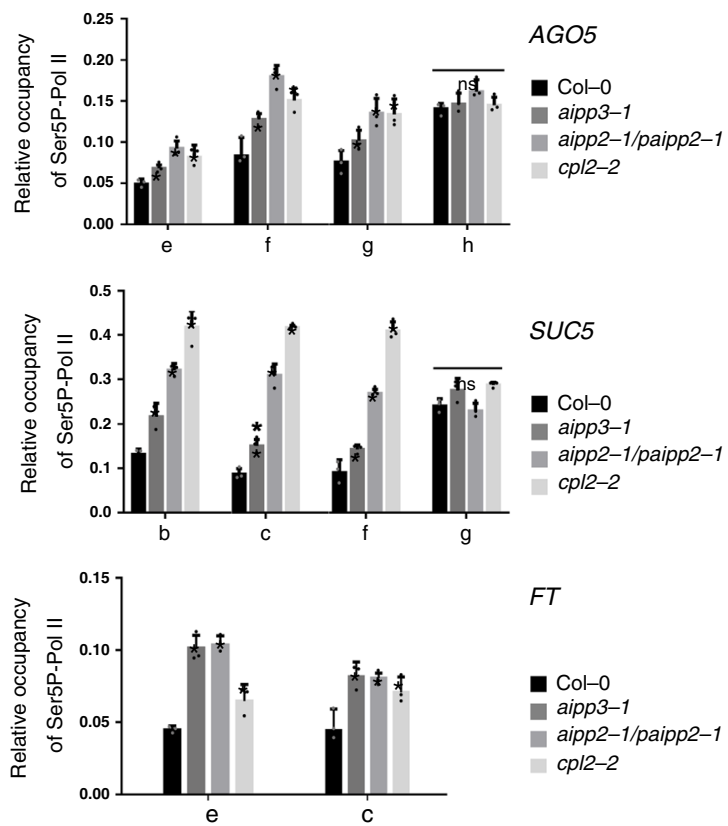

FT

- Col-0

aipp3-1

aipp2-1/paipp2-1

cpl2-2

C
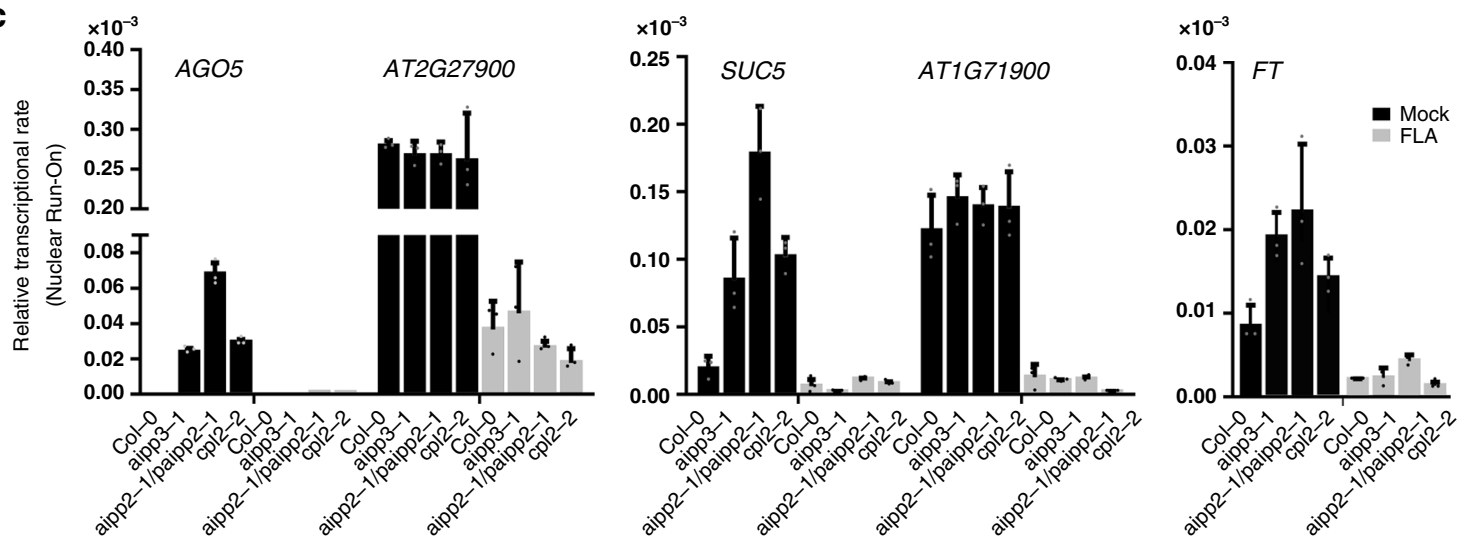

d
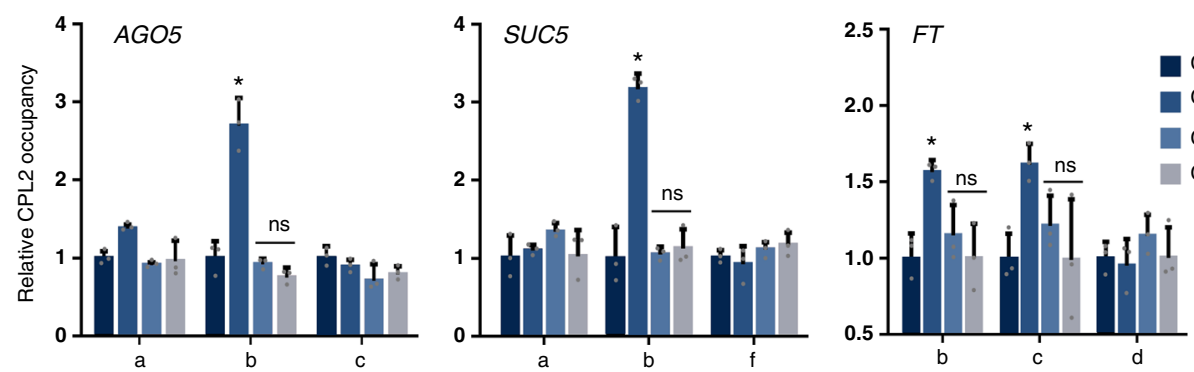

Col-0

CPL2-GFP/Col-0

CPL2-GFP/aipp3-1

CPL2-GFP/aipp2-1/paipp2-1

Fig. 7 BPC complex directly connects H3K27me3 recognition with transcriptional repression. a Snapshots showing the occupancies of different Pol II forms on selected target genes using a published Pol II NET-seq database. The red and blue lines indicate the Pol II signals in the plus and minus strands, respectively. The dashed red boxes indicate the occupancies of Pol II on the selected target genes. The black arrows indicate the transcriptional direction. b The ChIP-qPCR validation of the occupancy of Ser5P-Pol II on selected genes in different mutants. The lowercase letters the examined regions (the same below). The occupancy was normalized to the ACT7. The data are the means \pm S.D. of three biological repeats. Unpaired one-tailed $t$ test was performed and ${ }^{*} p$ value $<0.01$. ns no significance. $\mathbf{c}$ Nuclear run-on analysis showing the relative Pol II transcription rate at the selected target genes in Col- 0 and $b p c$ mutants with or without FLA treatment (mock). AT2G27900 and AT1G71900 genes serve as control genes. The relative transcription rate was normalized to $18 \mathrm{~S}$ rRNA. The data are the means \pm S.D. of three biological repeats. d CPL2 ChIP-qPCR results showing the relative occupancy of CPL2 at selected target genes in the presence and absence of AIPP3 and AIPP2/PAIPP2. The occupancy was first normalized to AtSN1 and then normalized to Col-0. The data are the means \pm S.D. of three biological repeats. Unpaired one-tailed $t$ test was performed and ${ }^{*} p$ value $<0.01$. 


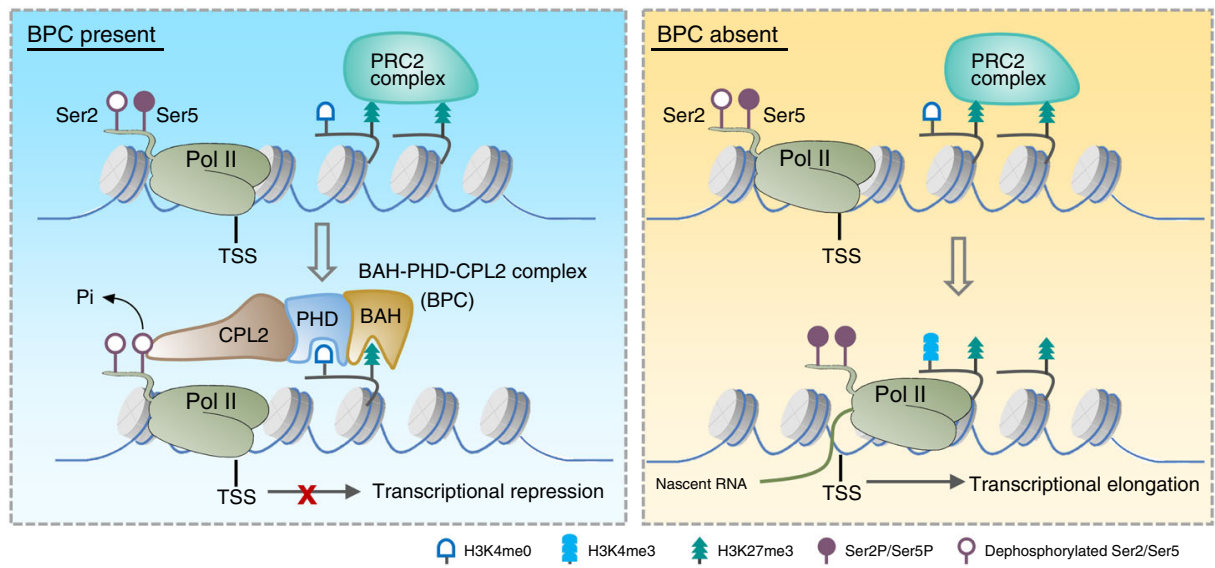

Fig. 8 A working model of the BPC complex-mediated transcription repression. When BPC is present, AIPP3-BAH, and AIPP2/PAIPP2-PHD motifs recognize H3K27me3, which is deposited by PRC2 complex, and unmodified H3K4 around the TSS, respectively. Then, the BAH-PHD histone reader module recruit CPL2 to dephosphorylate Pol II at the fifth Ser of CTD, thereby repressing the transcriptional initiation and subsequent elongation of Pol II. When BPC is absent, active H3K4me3 mark is deposited. Pol II CTD-Ser2 and -Ser5 residues can be phosphorylated sequentially, leading to release of Pol II from initiation to elongation state.

\section{Discussion}

Recently, we structurally described several plant H3K27me3reading $\mathrm{BAH}$ domain proteins, such as EBS, SHL, and AIPP3 in this research 27,28 , which has encouraged us to characterize the H3K27me3-reading BAH domains. The recognitions of $\mathrm{H} 3 \mathrm{~K} 27 \mathrm{me} 3$ by all these $\mathrm{BAH}$ domains depend on the aromatic cage to recognize the methyl-lysine, and a histidine and an aspartic acid residue to specifically interact with $\mathrm{H} 3 \mathrm{P} 30$ for sequence specificity. Using the aromatic cage, and the specific His and Asp residues as a criterion, we identified a subfamily of BAH domain proteins that can potentially recognize the $\mathrm{H} 3 \mathrm{~K} 27 \mathrm{me} 3$ mark, which are widely distributed in plants, fungi, and animals (Supplementary Fig. 13). Interestingly, the predicted H3K27me3binding $\mathrm{BAH}$ domain-containing protein human BAHD1 was reported to be an $\mathrm{H} 3 \mathrm{~K} 27 \mathrm{me} 3$ reader $^{30}$, which further supported our prediction. Therefore, we believe that the aromatic cage, and conserved $\mathrm{His}$ and Asp residues are the key features of H3K27me3-recognition BAH domains.

Different histone marks do not function in a totally independent manner. Instead, they engage in communications and cooperation with each other. The cooperation between different histone marks can be a combinatorial reading to enhance binding or be mutually exclusive to balance the binding between different marks, which occurs at both the single protein level and the multiple protein complex level ${ }^{68}$. Recently, we reported that the two flowering regulators EBS and SHL could dynamically recognize the antagonist histone marks $\mathrm{H} 3 \mathrm{~K} 27 \mathrm{me} 3$ and $\mathrm{H} 3 \mathrm{~K} 4 \mathrm{me} 3$ at the single protein level to regulate floral phase transitions ${ }^{27,28}$. Here, AIPP3 and AIPP2/PAIPP2 could recognize $\mathrm{H} 3 \mathrm{~K} 27 \mathrm{me} 3$ and $\mathrm{H} 3 \mathrm{~K} 4 \mathrm{me} 0$, respectively, and form a bivalent histone reader complex. This observation is consistent with our functional data showing that the BPC complex colocalizes with higher $\mathrm{H} 3 \mathrm{~K} 27 \mathrm{me} 3$ and lower $\mathrm{H} 3 \mathrm{~K} 4 \mathrm{me} 3$ marked chromatin regions. The BAH domain of AIPP3 is responsible for the colocalization of the complex with $\mathrm{H} 3 \mathrm{~K} 27 \mathrm{me} 3$. The binding of unmodified H3K4 by the PHD finger of AIPP2/PAIPP2 may have two roles. First, the binding of unmodified $\mathrm{H} 3 \mathrm{~K} 4$ may prevent the binding toward methylated $\mathrm{H} 3 \mathrm{~K} 4$ to make sure the complex is targeted to the gene repressive $\mathrm{H} 3 \mathrm{~K} 4 \mathrm{me} 3$ depletion region. Second, the binding of $\mathrm{H} 3 \mathrm{~K} 4 \mathrm{me} 0$ together with the $\mathrm{H} 3 \mathrm{~K} 27 \mathrm{me} 3$ binding by AIPP3 may combine to enhance the overall binding of the complex toward a certain chromatin region. Therefore, the proper targeting of the BPC complex relies on the crosstalk between the $\mathrm{H} 3 \mathrm{~K} 4 \mathrm{me} 0$ and $\mathrm{H} 3 \mathrm{~K} 27 \mathrm{me} 3$ marks.

RNA Pol II-dependent transcription is a stepwise process involving the formation of the preinitiation complex (PIC), initiation, elongation, and polyadenylation/termination stages. Each of the stages is associated with a distinct pattern of CTD phosphorylation $^{8,65}$. The transcriptional machinery is first recruited to the promoter regions. Once incorporated into the PIC, the mediator stimulates cyclin-dependent kinase to phosphorylate serine 5 of the CTD heptad repeat, and Ser5P helps in the release of Pol II from the PIC complex, thereby allowing Pol II to escape the promoter and the subsequent initiation of transcription. Ser5P is retained during the first several hundred nucleotides, in preparation for productive elongation ${ }^{69}$.

Here, on the selected target genes AGO5 and SUC5, unphosphorylated Pol II is restricted in the TSS region, whereas Ser5P and Ser2P were below detectable levels (Fig. 7a), indicating that Ser5P-dependent transcription initiation is inhibited, possibly by CPL2, in the presence of the BPC complex. Consistent with this notion, the density of the Ser5P-Pol II was significantly increased, and it peaked within the first several hundred nucleotides in the BPC complex mutants (Fig. 7b), indicating that the Ser5Pdependent transcription initiation was derepressed. In the canonical model of $\mathrm{H} 3 \mathrm{~K} 27 \mathrm{me} 3$-mediated transcription repression, the recognition of $\mathrm{H} 3 \mathrm{~K} 27 \mathrm{me} 3$ recruits the $\mathrm{PcG}$ proteins in the PRC1 complex to impose the monoubiquitination of $\mathrm{H} 2 \mathrm{~A}$, which represses transcription through three possible mechanisms, as mentioned in the introduction ${ }^{8}$. Consistent with this model, a recent report showed that the mutations in $\mathrm{H} 3 \mathrm{~K} 27 \mathrm{me} 3$ reader proteins LHP1, EBS, and SHL in Arabidopsis led to a reduction in H2Aub1 (ref. ${ }^{29}$ ). We found that the H2Aub1 levels were reduced in the lhp1-3 mutant, but were not affected in the aipp3-1/cpl2-2, aipp3-1/aipp2-1/paipp2-1, and aipp2-1/paipp2-1/cpl2-2 mutants (Supplementary Fig. 10a). This observation is consistent with our hypothesis that the BPC complex represses transcription mainly through the inhibition of Ser5P-dependent transcription initiation. While, we cannot rule out the possibility that BPC the complex has direct/indirect interaction with $\mathrm{H} 2 \mathrm{Aub} 1$ at specific target genes. This study unveiled a direct connection between $\mathrm{H} 3 \mathrm{~K} 27 \mathrm{me} 3$ recognition and transcription repression; the $\mathrm{BAH}-\mathrm{PHD}$ module recognizes $\mathrm{H} 3 \mathrm{~K} 27 \mathrm{me} 3 / \mathrm{H} 3 \mathrm{~K} 4 \mathrm{me} 0$ and recruits CPL2 to dephosphorylate Pol II CTD, resulting in the 
failure of Pol II to enter into the initiation and elongation form. Although Pol II CTD phosphatases are conserved in eukaryotes, no evidence shows that histone modifiers manipulate CTD phosphatases, including CPL2 analogies in other systems, to affect transcription. Regarding histone modifiers, several studies have reported that histone PTM modifiers can affect transcription via

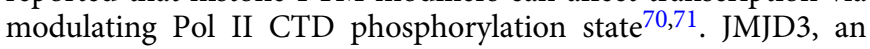
H3K27me3 demethylase in human, has been shown to directly interact with CTD-Ser2P to affect gene expression ${ }^{72}$. Knocking down JMJD3 or JHDM1D, a H3K27me1/2 demethylase, reduces the enrichment of Pol II CTD-Ser2P at specific genes in human promyelocytic leukemia cells ${ }^{73}$. In addition to histone methylation, other histone PTMs including histone ubiquitylation and phosphorylation, are also associated with Pol II CTD phosphorylation-dependent transcriptional elongation ${ }^{71}$. For example, knocking down histone ubiquitylation modifiers has been shown to affect CTD-Ser2 phosphorylation ${ }^{71}$. The phosphorylation of histone $\mathrm{H} 3$ on S10 and S28 has been reported to be associated with phosphorylated Pol II during transcriptional activation in humans and Drosophila ${ }^{74,75}$. These studies support a notion that modulation of Pol II CTD phosphorylation represents an important regulatory mechanism adopted by chromatin regulators to regulate gene expression. Our finding that the BPC complex reading histone information and conferring transcriptional repression through CPL2 phosphatase-mediated modulation of Pol II CTD phosphorylation state provides a direct evidence to support this notion. Considering that CPL2 is a plantspecific Pol II phosphatase that bears a unique RBM domain, and that this domain is required for its interaction with PHD proteins (Fig. 1c), the BAH-PHD-CPL2 pathway may represent a newly evolved silencing pathway in plants. Our findings suggest a greater complexity and diversity of H3K27me3-mediated transcriptional regulation. In addition, it is well-known that H3K27me3-mediated silencing mechanisms participate in multiple biological processes in both plants and animals, particularly development and stress-responsive genes. The obvious developmental defects (Fig. 2a) and reactivation of many developmentand stress response-related genes (Supplementary Fig. 9) in $b p c$ mutants imply that the BPC complex may play more important roles in plant development and stress responses in addition to flowering time control.

\section{Methods}

Plant materials and growth conditions. All the plant seeds were sown and grown on $1 / 2$ MS medium containing $1 \%$ sucrose. The seedlings were grown under a LD ( $16 \mathrm{~h}$ light $/ 8 \mathrm{~h}$ dark) or SD ( $8 \mathrm{~h}$ light $/ 16 \mathrm{~h}$ dark) photoperiod at $23^{\circ} \mathrm{C}$. The T-DNA insertion mutants aipp2-1, aipp3-1, and $c p l 2-2$ were described in our previous study ${ }^{41}$. The paipp 2-1 mutant was generated by CRISPR/Cas9-mediated mutagenesis in a Col-0 background (Supplementary Fig. 4). clf-81 and lhp1-3 have been described previously 53,54 . For the epitope-tagged transgenic expression of the AIPP2, AIPP3, PAIPP2, and CPL2 genes, the wild-type and mutated genomic DNA driven by their native promoters were cloned into binary vectors with different tags and then transformed into the corresponding mutants, using the flowering dip method. T3 generation transgenic plants were used for analysis.

FLA treatment assay was performed as previous report ${ }^{67}$. In brief, 2-week-old seedlings were collected and incubated with $200 \mu \mathrm{M}$ FLA or mock solution (DMSO) overnight. The treated seedlings were subjected to total RNA extraction.

RT-qPCR and RNA-seq analysis. Total RNA was extracted from 2-week-old seedlings using Trizol reagent (Thermo), and CDNAs were synthesized using HiScript II Reverse Transcriptase (Vazyme). qPCR was performed using a CFX96 Touch Deep Well Real-Time PCR Detection System (Bio-Rad). Three biological replicates were created. The primers used in this study are listed in Supplementary Table 2. For the RNA-seq analysis, total RNAs were extracted from 12-day-old seedlings grown during LDs using a RNeasy Plant Mini kit (Qiagen). Following RNA purification, reverse transcription and library construction, the libraries were quantified by TBS380, and a paired-end RNA sequencing library was performed with Illumina NovaSeq $6000(2 \times 150 \mathrm{bp}$ read length). The raw paired-end reads were trimmed and subject to quality control with SeqPrep (https://github.com/
jstjohn/SeqPrep) and Sickle (https://github.com/najoshi/sickle), using the default parameters.

IP and MS analysis. IP and MS analyses were performed as previously described ${ }^{76}$ In brief, the total proteins were extracted from the inflorescence tissues with IP buffer (50 mM Tris- $\mathrm{HCl}, \mathrm{pH} 7.6,150 \mathrm{mM} \mathrm{NaCl}, 5 \mathrm{mM} \mathrm{MgCl} 2,10 \%$ glycerol, $0.1 \%$ NP-40, $0.5 \mathrm{mM}$ DTT, and protease inhibitor cocktail), and then precipitated with anti-Flag (Sigma-Aldrich) or anti-Myc (Millipore) antibodies for $2 \mathrm{~h}$ at $4{ }^{\circ} \mathrm{C}$. After five times washing, the precipitated protein mixtures were subjected to MS analysis.

Protein interaction analysis and gel filtration assays. For the $\mathrm{Y} 2 \mathrm{H}$ assays, the full-length and truncated coding sequences of AIPP2, AIPP3, CPL2, and PAIPP2 were cloned into the pGADT7 and pGBKT7 vectors to generate $\mathrm{AD}$ and $\mathrm{BD}$ constructs. After the transformation, the yeast cultures were spotted onto SD plates lacking Trp and Leu (-LW) or lacking Trp, Leu, and His (-LWH) and incubated at $30{ }^{\circ} \mathrm{C}$ for $3 \mathrm{~d}$.

For gel filtration assay, the total proteins were extracted from $4 \mathrm{~g}$ of seedling tissues expressing AIPP3-FLAG, AIPP2-4MYC, PAIPP2-3FLAG, and CPL2-4MYC with IP buffer and then loaded on to a Superdex 200 10/300GL column (GE Healthcare). The eluted fractions were collected in 96-well plates and the target proteins were detected by standard western blotting.

Protein expression and purification. The sequence containing the AIPP3-BAH domain (residues 112-279) was constructed into a self-modified pMal-p2X vector to fuse a hexahistidine tag plus a maltose-binding protein (MBP) tag to the Nterminus of the target protein. The plasmids were transformed into Escherichia coli strain BL21 (DE3) RIL (Stratagene). Expression was induced at $16^{\circ} \mathrm{C}$ overnight with $0.2 \mathrm{mM}$ of IPTG. The recombinant proteins were purified with a prepackaged HisTrap FF column (GE Healthcare). The His-MBP tags were cleaved by TEV protease overnight and removed by flowing through a HisTrap FF column (GE Healthcare) again. The target protein was further purified using a Heparin column (GE Healthcare) and a Superdex G200 column (GE Healthcare). All the AIPP3BAH mutations were generated by standard PCR-based mutagenesis procedure. The mutations of AIPP3-BAH and truncated AIPP2/PAIPP2 fragments were purified using the same protocols, as those used for the wild-type AIPP3-BAH. For the GST-AIPP3-BAH proteins used in histone peptide pull-down assays, the wildtype and mutated AIPP3-BAH proteins were purified with glutathione-Sepharose (GE Healthcare) and eluted with elution buffer $(50 \mathrm{mM}$ Tris- $\mathrm{HCl} \mathrm{pH} \mathrm{8.0,} \mathrm{and}$ $10 \mathrm{mM}$ reduced glutathione). The peptides were purchased from GL Biochem or EpiCypher.

Histone peptide pull-down. For histone peptide pull-down assay, $1.5 \mu \mathrm{g}$ of biotinylated histone peptides were incubated with streptavidin beads (NEB) in binding buffer $(50 \mathrm{mM}$ Tris- $\mathrm{HCl} 8.0,300 \mathrm{mM} \mathrm{NaCl}$, and $0.1 \% \mathrm{NP}-40)$ for $1 \mathrm{~h}$ at $4{ }^{\circ} \mathrm{C}$, and then washed with binding buffer. A $1.5 \mu \mathrm{g}$ quantity of AIPP3-BAH proteins was incubated with a peptide-bead mixture in $0.5 \mathrm{ml}$ of binding buffer for $3 \mathrm{~h}$ at $4{ }^{\circ} \mathrm{C}$. and then washed with binding buffer five times. The protein-bead mixtures were subjected to immunoblotting using anti-GST antibody (Abmart, \#12G8, 1:2000 dilution).

\section{Chromatin immunoprecipitation assays and ChIP-seq analysis. ChIP assays} were performed according to a reported procedure ${ }^{77}$. In brief, $3 \mathrm{~g}$ of seedlings was harvested and fixed with $1 \%$ formaldehyde in the cross-linking buffer $(0.4 \mathrm{M}$ sucrose, $10 \mathrm{mM}$ Tris- $\mathrm{HCl} \mathrm{pH} 8,1 \mathrm{mM}$ PMSF, and $1 \mathrm{mM}$ EDTA). After nuclei isolation with isolation buffer $\left(0.25 \mathrm{M}\right.$ sucrose, $15 \mathrm{mM}$ PIPES pH $6.8,5 \mathrm{mM} \mathrm{MgCl}_{2}$, $60 \mathrm{mM} \mathrm{KCl}, 15 \mathrm{mM} \mathrm{NaCl}, 1 \mathrm{mM} \mathrm{CaCl}_{2}, 0.9 \%$ Triton X-100, and protease inhibitor cocktail) and the following centrifugation, nuclei were resuspended in $500 \mu \mathrm{l}$ of lysis buffer (50 mM HEPES pH 7.5, $150 \mathrm{mM} \mathrm{NaCl}, 1 \mathrm{mM}$ EDTA, $1 \mathrm{mM}$ PMSF, 1\% SDS, $0.1 \%$ Na deoxycholate, $1 \%$ Triton X-100, and protease inhibitor cocktail) and sonicated with a Bioruptor (Diagenode). The nuclei lysate was precipitated with anti-Flag (Sigma-Aldrich, \#F1804, 1:100 dilution), anti-Myc (Millipore, \#05-724, 1:100 dilution), anti-H3 (Abcam, \#ab1791, 1:100 dilution), anti-H3K27me3 (Millipore, \#07-449, 1:100 dilution), Ser2P-Pol II (Abcam, \#ab5095, 1:100 dilution), Ser5P-Pol II (Abcam, \#ab5131, 1:100 dilution), unphosphorylated Pol II (Abcam, \#ab817, 1:100 dilution), and GFP (Abcam, \#ab290, 1:100 dilution) antibodies overnight and incubated with Dynabeads (Thermo) for $2 \mathrm{~h}$. The precipitated protein-DNA mixtures were washed and eluted with elution buffer $(0.5 \%$ SDS and $0.1 \mathrm{M} \mathrm{NaHCO} 3)$ at room temperature. The DNA was recovered after reverse crosslinking and proteinase $\mathrm{K}$ treatment.

For ChIP-seq analysis, clean reads were mapped to the Arabidopsis thaliana genome (TAIR10) by Bowtie2 (version 2.2.8) with default parameters ${ }^{78}$. Enriched peaks were identified by MACS (version 1.4) with default parameters. We defined the region of a target gene as the range from $1 \mathrm{~kb}$ upstream of TSS to TTS. The target genes of each peak were annotated by annotatePeak function in ChIPseeker package. The visualization of the average read coverage over gene body and additional $1 \mathrm{~kb}$ upstream and downstream of the TSS and TES was performed by deepTools (version 2.4.1) ${ }^{79}$. 
Crystallization, data collection. and structure determination. Crystallization screening was performed using the sitting drop vapor diffusion method at $4{ }^{\circ} \mathrm{C}$. The sample was concentrated and mixed with peptide at a molar ratio of 1:4. All the crystals emerged in a solution of 0.1 M HEPES, $\mathrm{pH} 7.0$, and $2.4 \mathrm{M}$ ammonium sulfate. The crystals were cryo-protected in the reservoir solution supplemented with $20 \%$ glycerol and flash-cooled in liquid nitrogen for X-ray diffraction. All the diffraction data were collected at beamline BL19U1 of the National Center for Protein Sciences Shanghai (NCPSS) at the Shanghai Synchrotron Radiation Facility (SSRF). The data were processed with the HKL3000 program $^{80}$. The structure was determined by molecular replacement method as implemented in the Phenix program ${ }^{81}$, using the ZMET2 BAH domain (PDB ID: 4FT2) as the searching mode ${ }^{20}$. Model building and structure refinement were performed using the Coot and Phenix programs, respectively ${ }^{81,82}$. The statistics of data collection and structure refinement are listed in Supplementary Table 1 .

ITC calorimetry. All the binding experiments were performed on a Microcal PEAQ-ITC instrument (Malvern) at $25^{\circ} \mathrm{C}$. The proteins were dialyzed against a buffer consisting of $50 \mathrm{mM} \mathrm{NaCl}$ and $20 \mathrm{mM}$ Tris- $\mathrm{HCl}$, pH 7.5 overnight at $4{ }^{\circ} \mathrm{C}$. The lyophilized peptides were dissolved into the dialysis buffer. The titration was performed using the standard protocol and the data were processed using the Origin 7.0 program.

Nuclear run-on assay. Nuclear run-on assay was performed as previous report with minor changes ${ }^{83}$. In brief, after nuclei isolation with buffer $(0.25 \mathrm{M}$ sucrose, $15 \mathrm{mM}$ PIPES pH 6.8, $5 \mathrm{mM} \mathrm{MgCl}_{2}, 60 \mathrm{mM} \mathrm{KCl}, 15 \mathrm{mM} \mathrm{NaCl}, 1 \mathrm{mM} \mathrm{CaCl} 2,0.9 \%$ Triton X-100, and protease inhibitor cocktail), the nuclear pellet was resuspended with nuclei storage buffer (50 mM Tris- $\mathrm{HCl} \mathrm{pH} 8,0.1 \mathrm{mM}$ EDTA, $5 \mathrm{mM} \mathrm{MgCl}_{2}$ and $40 \%$ glycerol) and mixed with transcription buffer ( $10 \mathrm{mM}$ Tris- $\mathrm{HCl}, \mathrm{pH} 8$, $2.5 \mathrm{mM} \mathrm{MgCl}_{2}, 150 \mathrm{mM} \mathrm{KCl}, 2 \mathrm{mM}$ DTT, 80 units RNase inhibitor, $0.5 \mathrm{mM}$ BrUTP, $1 \mathrm{mM}$ ATP, $1 \mathrm{mM}$ GTP, $1 \mathrm{mM}$ CTP, $0.5 \mathrm{mM}$ UTP, and $0.2 \%$ sarkosyl). After incubation at $30^{\circ} \mathrm{C}$ for $15 \mathrm{~min}$, the run-on reaction was stopped by adding Trizol reagent (Thermo). The nuclear RNAs were extracted and treated with DNase. The DNA-free RNAs were then inoculated with $2 \mu \mathrm{g}$ anti-BrdU antibody (abcam) at room temperature for $30 \mathrm{~min}$ and precipitated by Dynabeads (Thermo). After two times washing, RNAs were extracted using Trizol reagent (Thermo). cDNAs were synthesized using SuperScript IV Reverse Transcriptase (Thermo) and subjected to qPCR analysis.

GUS staining assay. The plant materials were stained in staining solution overnight at $37^{\circ} \mathrm{C}$ and then washed with $70 \%$ ethanol at room temperature. The plants were cleared after being washed and were observed under a ZEISS Stemi 305 microscope.

Histone immunoblotting. The nuclei were extracted from 2-week-old seedlings with isolation buffer $\left(0.25 \mathrm{M}\right.$ sucrose, $15 \mathrm{mM}$ PIPES pH $6.8,5 \mathrm{mM} \mathrm{MgCl}_{2}, 60 \mathrm{mM}$ $\mathrm{KCl}, 15 \mathrm{mM} \mathrm{NaCl}, 1 \mathrm{mM} \mathrm{CaCl} 2,0.9 \%$ Triton X-100, and protease inhibitor cocktail) and resuspended with lysis buffer (50 mM HEPES pH 7.5, $150 \mathrm{mM} \mathrm{NaCl}$, $1 \mathrm{mM}$ EDTA, $1 \mathrm{mM}$ PMSF, $1 \%$ SDS, $0.1 \%$ Na deoxycholate, $1 \%$ Triton X-100, and protease inhibitor cocktail) to release the total chromatin. The supernatant was boiled with SDS loading buffer and subjected to immunoblotting with anti-H3 (Abcam, \#ab1791, 1:1000 dilution), anti-H3K27me1 (Millipore, \#07-448, 1:1000 dilution), H3K27me2 (Millipore, \#07-452, 1:1000 dilution), H3K27me3 (Millipore, \#07-449, 1:1000 dilution), anti-H3K4me1 (Millipore, \#07-436, 1:1000 dilution), anti-H3K4me2 (Millipore, \#07-030, 1:1000 dilution), anti-H3K4me3 (Millipore, \#07-473, 1:1000 dilution), and anti-H2AKub (Cell Signaling, \#8240, 1:1000 dilution) antibodies.

Split luciferase assay. For split luciferase assay, the constructs fused with split luciferase were co-infiltrated into tobacco (Nicotiana benthamiana) leaves. The luciferase activity was determined at 2 days after infiltration using CCD camera (Princeton instruments)

Reporting summary. Further information on research design is available in the Nature Research Reporting Summary linked to this article.

\section{Data availability}

Coordinates and structure factors have been deposited in the RCSB Protein Data Bank with the accession code: 7CCE. The ChIP-seq and mRNA-seq data have been deposited in the GEO with the accession codes GSE147981 and GSE157196, respectively. All other data are available from the corresponding authors on request. Source data are provided with this paper.

Received: 22 April 2020; Accepted: 12 November 2020; Published online: 04 December 2020

\section{References}

1. Kim, S. Y., Lee, J., Eshed-Williams, L., Zilberman, D. \& Sung, Z. R. EMF1 and PRC2 cooperate to repress key regulators of Arabidopsis development. PLoS Genet. 8, e1002512 (2012).

2. Strahl, B. D. \& Allis, C. D. The language of covalent histone modifications. Nature 403, 41-45 (2000).

3. Campos, E. I. \& Reinberg, D. Histones: annotating chromatin. Annu Rev. Genet. 43, 559-599 (2009).

4. Liu, C., Lu, F., Cui, X. \& Cao, X. Histone methylation in higher plants. Annu. Rev. Plant Biol. 61, 395-420 (2010)

5. Chang, Y. N. et al. Epigenetic regulation in plant abiotic stress responses. J. Integr. Plant Biol. 62, 563-580 (2019).

6. Kim, D. H. \& Sung, S. Polycomb-mediated gene silencing in Arabidopsis thaliana. Molecules Cells 37, 841-850 (2014).

7. Simon, J. A. \& Kingston, R. E. Mechanisms of polycomb gene silencing: knowns and unknowns. Nat. Rev. Mol. Cell Biol. 10, 697-708 (2009).

8. Aranda, S., Mas, G. \& Di Croce, L. Regulation of gene transcription by Polycomb proteins. Sci. Adv. 1, e1500737 (2015).

9. Di Croce, L. \& Helin, K. Transcriptional regulation by Polycomb group proteins. Nat. Struct. Mol. Biol. 20, 1147-1155 (2013).

10. Blackledge, N. P., Rose, N. R. \& Klose, R. J. Targeting Polycomb systems to regulate gene expression: modifications to a complex story. Nat. Rev. Mol. Cell Biol. 16, 643-649 (2015).

11. Shao, Z. et al. Stabilization of chromatin structure by PRC1, a Polycomb complex. Cell 98, 37-46 (1999).

12. Levine, S. S. et al. The core of the polycomb repressive complex is compositionally and functionally conserved in flies and humans. Mol. Cell. Biol. 22, 6070-6078 (2002).

13. Francis, N. J., Kingston, R. E. \& Woodcock, C. L. Chromatin compaction by a polycomb group protein complex. Science 306, 1574-1577 (2004).

14. Chopra, V. S. et al. The polycomb group mutant esc leads to augmented levels of paused Pol II in the Drosophila embryo. Mol. Cell 42, 837-844 (2011).

15. Zhou, W. et al. Histone $\mathrm{H} 2 \mathrm{~A}$ monoubiquitination represses transcription by inhibiting RNA polymerase II transcriptional elongation. Mol. Cell 29, 69-80 (2008).

16. Stock, J. K. et al. Ring1-mediated ubiquitination of $\mathrm{H} 2 \mathrm{~A}$ restrains poised RNA polymerase II at bivalent genes in mouse ES cells. Nat. Cell Biol. 9, 1428-1435 (2007).

17. Liu, R., Li, X., Chen, W. \& Du, J. Structure and mechanism of plant histone mark readers. Sci. China Life Sci. 61, 170-177 (2018).

18. Yang, N. \& Xu, R. M. Structure and function of the BAH domain in chromatin biology. Crit. Rev. Biochem. Mol. Biol. 48, 211-221 (2013).

19. Li, Y. \& Li, H. Many keys to push: diversifying the 'readership' of plant homeodomain fingers. Acta Biochim. Biophys. Sin. 44, 28-39 (2012).

20. Du, J. et al. Dual binding of chromomethylase domains to H3K9me2containing nucleosomes directs DNA methylation in plants. Cell 151, 167-180 (2012).

21. Kuo, A. J. et al. The BAH domain of ORC1 links H4K20me2 to DNA replication licensing and Meier-Gorlin syndrome. Nature 484, 115-119 (2012).

22. Li, S. et al. Structural basis for the unique multivalent readout of unmodified H3 tail by Arabidopsis ORC1b BAH-PHD cassette. Structure 24, 486-494 (2016).

23. Armache, K. J., Garlick, J. D., Canzio, D., Narlikar, G. J. \& Kingston, R. E. Structural basis of silencing: Sir3 BAH domain in complex with a nucleosome at 3.0 A resolution. Science 334, 977-982 (2011)

24. Yang, D. et al. Nalpha-acetylated Sir3 stabilizes the conformation of a nucleosome-binding loop in the BAH domain. Nat. Struct. Mol. Biol. 20, 1116-1118 (2013).

25. Arnaudo, N. et al. The N-terminal acetylation of Sir3 stabilizes its binding to the nucleosome core particle. Nat. Struct. Mol. Biol. 20, 1119-1121 (2013).

26. Wang, F. et al. Heterochromatin protein Sir3 induces contacts between the amino terminus of histone $\mathrm{H} 4$ and nucleosomal DNA. Proc. Natl Acad. Sci. USA 110, 8495-8500 (2013).

27. Yang, Z. et al. EBS is a bivalent histone reader that regulates floral phase transition in Arabidopsis. Nat. Genet. 50, 1247-1253 (2018).

28. Qian, S. et al. Dual recognition of H3K4me3 and H3K27me3 by a plant histone reader SHL. Nat. Commun. 9, 2425 (2018).

29. Li, Z., Fu, X., Wang, Y., Liu, R. \& He, Y. Polycomb-mediated gene silencing by the BAH-EMF1 complex in plants. Nat. Genet. 50, 1254-1261 (2018).

30. Zhao, D. et al. The BAH domain of BAHD1 is a histone $\mathrm{H} 3 \mathrm{~K} 27$ me3 reader. Protein Cell 7, 222-226 (2016).

31. Luo X. \& He Y. Experiencing winter for spring flowering: a molecular epigenetic perspective on vernalization. J. Integr. Plant Biol. 62, 104-117 (2019).

32. Noh, B. et al. Divergent roles of a pair of homologous jumonji/zinc-fingerclass transcription factor proteins in the regulation of Arabidopsis flowering time. Plant Cell 16, 2601-2613 (2004). 
33. Goodrich, J. et al. A Polycomb-group gene regulates homeotic gene expression in Arabidopsis. Nature 386, 44-51 (1997).

34. Yan, W. et al. Dynamic and spatial restriction of Polycomb activity by plant histone demethylases. Nat. Plants 4, 681-689 (2018).

35. Zheng, S. et al. The Arabidopsis H3K27me3 demethylase JUMONJI 13 is a temperature and photoperiod dependent flowering repressor. Nat. Commun. 10, 1303 (2019).

36. Yang, H., Howard, M. \& Dean, C. Physical coupling of activation and derepression activities to maintain an active transcriptional state at FLC. Proc. Natl Acad. Sci. USA 113, 9369-9374 (2016).

37. Gaudin, V. et al. Mutations in LIKE HETEROCHROMATIN PROTEIN 1 affect flowering time and plant architecture in Arabidopsis. Development 128, 4847-4858 (2001).

38. Gomez-Mena, C. et al. early bolting in short days: an Arabidopsis mutation that causes early flowering and partially suppresses the floral phenotype of leafy. Plant Cell 13, 1011-1024 (2001).

39. Sung, S. et al. Epigenetic maintenance of the vernalized state in Arabidopsis thaliana requires LIKE HETEROCHROMATIN PROTEIN 1. Nat. Genet. 38, 706-710 (2006).

40. Mylne, J. S. et al. LHP1, the Arabidopsis homologue of HETEROCHROMATIN PROTEIN1, is required for epigenetic silencing of FLC. Proc. Natl Acad. Sci. USA 103, 5012-5017 (2006).

41. Duan, C. G. et al. A protein complex regulates RNA processing of intronic heterochromatin-containing genes in Arabidopsis. Proc. Natl Acad. Sci. USA 114, E7377-E7384 (2017).

42. Wang, X. et al. RNA-binding protein regulates plant DNA methylation by controlling mRNA processing at the intronic heterochromatin-containing gene IBM1. Proc. Natl Acad. Sci. USA 110, 15467-15472 (2013).

43. Michaels, S. D. \& Amasino, R. M. FLOWERING LOCUS C encodes a novel MADS domain protein that acts as a repressor of flowering. Plant Cell 11, 949-956 (1999).

44. Sheldon, C. C. et al. The FLF MADS box gene: a repressor of flowering in Arabidopsis regulated by vernalization and methylation. Plant Cell 11, 445-458 (1999).

45. Searle, I. et al. The transcription factor FLC confers a flowering response to vernalization by repressing meristem competence and systemic signaling in Arabidopsis. Genes Dev. 20, 898-912 (2006).

46. Suarez-Lopez, P. et al. CONSTANS mediates between the circadian clock and the control of flowering in Arabidopsis. Nature 410, 1116-1120 (2001).

47. Imaizumi, T. \& Kay, S. A. Photoperiodic control of flowering: not only by coincidence. Trends Plant Sci. 11, 550-558 (2006).

48. Turck, F., Fornara, F. \& Coupland, G. Regulation and identity of florigen FLOWERING LOCUS T moves center stage. Annu. Rev. Plant Biol. 59, 573-594 (2008).

49. Dennis, E. S. \& Peacock, W. J. Epigenetic regulation of flowering. Curr. Opin. Plant Biol. 10, 520-527 (2007).

50. Duan, C. G., Zhu, J. K. \& Cao, X. Retrospective and perspective of plant epigenetics in China. J. Genet. Genomics 45, 621-638 (2018).

51. Patel, D. J. A structural perspective on readout of epigenetic histone and DNA methylation marks. Cold Spring Harb. Perspect. Biol. 8, a018754 (2016).

52. Bergamin, E. et al. Molecular basis for the methylation specificity of ATXR5 for histone H3. Nucleic Acids Res. 45, 6375-6387 (2017).

53. Kim, G. T., Tsukaya, H. \& Uchimiya, H. The CURLY LEAF gene controls both division and elongation of cells during the expansion of the leaf blade in Arabidopsis thaliana. Planta 206, 175-183 (1998).

54. Lindroth, A. M. et al. Dual histone $\mathrm{H} 3$ methylation marks at lysines 9 and 27 required for interaction with CHROMOMETHYLASE3. EMBO J. 23, 4286-4296 (2004).

55. Takagi, J. et al. MAIGO5 functions in protein export from Golgi-associated endoplasmic reticulum exit sites in Arabidopsis. Plant Cell 25, 4658-4675 (2013).

56. Pastor-Cantizano, N., Bernat-Silvestre, C., Marcote, M. J. \& Aniento F. Loss of Arabidopsis p24 function affects ERD2 trafficking and Golgi structure, and activates the unfolded protein response. J. Cell Sci. 131, jcs203802 (2018).

57. Gimeno-Ferrer, F. et al. alpha2-COP is involved in early secretory traffic in Arabidopsis and is required for plant growth. J. Exp. Bot. 68, 391-401 (2017).

58. Brosseau, C. \& Moffett, P. Functional and genetic analysis identify a role for Arabidopsis ARGONAUTE5 in antiviral RNA silencing. Plant Cell 27, 1742-1754 (2015).

59. Garcia-Ruiz, H. et al. Roles and programming of Arabidopsis ARGONAUTE proteins during Turnip mosaic virus infection. PLoS Pathog. 11, e1004755 (2015).

60. Minoia, S. et al. Specific argonautes selectively bind small RNAs derived from potato spindle tuber viroid and attenuate viroid accumulation in vivo. J. Virol. 88, 11933-11945 (2014).

61. Tucker, M. R. et al. Somatic small RNA pathways promote the mitotic events of megagametogenesis during female reproductive development in Arabidopsis. Development 139, 1399-1404 (2012).
62. Borges, F., Pereira, P. A., Slotkin, R. K., Martienssen, R. A. \& Becker, J. D. MicroRNA activity in the Arabidopsis male germline. J. Exp. Bot. 62, $1611-1620$ (2011)

63. Luo, C. et al. Integrative analysis of chromatin states in Arabidopsis identified potential regulatory mechanisms for natural antisense transcript production. Plant J. 73, 77-90 (2013).

64. Harlen, K. M. \& Churchman, L. S. The code and beyond: transcription regulation by the RNA polymerase II carboxy-terminal domain. Nat. Rev. Mol. Cell Biol. 18, 263-273 (2017).

65. Hajheidari, M., Koncz, C. \& Eick, D. Emerging roles for RNA polymerase II CTD in Arabidopsis. Trends Plant Sci. 18, 633-643 (2013).

66. Koiwa, H. et al. Arabidopsis C-terminal domain phosphatase-like 1 and 2 are essential Ser-5-specific C-terminal domain phosphatases. Proc. Natl Acad. Sci. USA 101, 14539-14544 (2004).

67. Zhu, J., Liu, M., Liu, X. \& Dong, Z. RNA polymerase II activity revealed by GRO-seq and pNET-seq in Arabidopsis. Nat. Plants 4, 1112-1123 (2018).

68. Du, J. \& Patel, D. J. Structural biology-based insights into combinatorial readout and crosstalk among epigenetic marks. Biochim. Biophys. Acta 1839, 719-727 (2014).

69. Ding, Y., Avramova, Z. \& Fromm, M. Two distinct roles of ARABIDOPSIS HOMOLOG OF TRITHORAX1 (ATX1) at promoters and within transcribed regions of ATX1-regulated genes. Plant Cell 23, 350-363 (2011).

70. Kooistra, S. M. \& Helin, K. Molecular mechanisms and potential functions of histone demethylases. Nat. Rev. Mol. Cell Biol. 13, 297-311 (2012).

71. Srivastava, R. \& Ahn, S. H. Modifications of RNA polymerase II CTD: connections to the histone code and cellular function. Biotechnol. Adv. 33, 856-872 (2015).

72. Estaras, C., Fueyo, R., Akizu, N., Beltran, S. \& Martinez-Balbas, M. A. RNA polymerase II progression through $\mathrm{H} 3 \mathrm{~K} 27 \mathrm{me} 3$-enriched gene bodies requires JMJD3 histone demethylase. Mol. Biol. Cell 24, 351-360 (2013).

73. Chen, S. et al. The histone H3 Lys 27 demethylase JMJD3 regulates gene expression by impacting transcriptional elongation. Genes Dev. 26, 1364-1375 (2012).

74. Ivaldi, M. S., Karam, C. S. \& Corces, V. G. Phosphorylation of histone H3 at Ser10 facilitates RNA polymerase II release from promoter-proximal pausing in Drosophila. Genes Dev. 21, 2818-2831 (2007).

75. Rossetto, D., Avvakumov, N. \& Cote, J. Histone phosphorylation: a chromatin modification involved in diverse nuclear events. Epigenetics 7, 1098-1108 (2012).

76. Duan, C. G. et al. A pair of transposon-derived proteins function in a histone acetyltransferase complex for active DNA demethylation. Cell Res. 27, 226-240 (2017).

77. Saleh, A., Alvarez-Venegas, R. \& Avramova, Z. An efficient chromatin immunoprecipitation (ChIP) protocol for studying histone modifications in Arabidopsis plants. Nat. Protoc. 3, 1018-1025 (2008).

78. Langmead, B. \& Salzberg, S. L. Fast gapped-read alignment with Bowtie 2. Nat. Methods 9, 357-359 (2012).

79. Ramirez, F. et al. deepTools2: a next generation web server for deepsequencing data analysis. Nucleic Acids Res. 44, W160-W165 (2016).

80. Otwinowski, Z. \& Minor, W. Processing of X-ray diffraction data collected in oscillation mode. Methods Enzymol. 276, 307-326 (1997).

81. Adams, P. D. et al. PHENIX: a comprehensive Python-based system for macromolecular structure solution. Acta Crystallogr. Sect. D. Biol. Crystallogr. 66, 213-221 (2010)

82. Emsley, P., Lohkamp, B., Scott, W. G. \& Cowtan, K. Features and development of Coot. Acta Crystallogr. Sect. D. Biol. Crystallogr. 66, 486-501 (2010).

83. Roberts, T. C. et al. Quantification of nascent transcription by bromouridine immunocapture nuclear run-on RT-qPCR. Nat. Protoc. 10, 1198-1211 (2015).

\section{Acknowledgements}

We thank Dr. Yuehui He for the seeds of $c l f-81, \operatorname{lhp} 1-3$, and $f l c-3$ mutants. We thank staff at beamline BL19U1 of the National Center for Protein Sciences Shanghai (NCPSS) at the Shanghai Synchrotron Radiation Facility (SSRF) for data collection. C.-G.D. was supported by the Strategic Priority Research Program of the Chinese Academy of Sciences (XDB27040203) and by the National Natural Science Foundation of China (31570155). J.-K.Z. was supported by the Strategic Priority Research Program of the Chinese Academy of Sciences(XDB2704). J.D. was supported by the National Natural Science Foundation of China (31770782), Shenzhen Science and Technology Program (JCYJ20200109110403829 and KQTD20190929173906742), SUSTech (G02226301), and the Key Laboratory of Molecular Design for Plant Cell Factory of Guangdong Higher Education Institutes (2019KSYS006).

\section{Author contributions}

C.-G.D., J.D., and J.-K.Z. designed this study. Y.-Z.Z., J.Y., L.Z., Y.W., G.Z., and S.-S.X. conducted the experiments. Y.Z.Z., J.Y., L.Z., J.J., J.D., J.-K.Z., and C.-G.D. analyzed the 
data. C.C. and P.L. performed bioinformatic analysis. J.D., J.-K.Z., and C.-G.D. wrote the paper.

\section{Competing interests}

The authors declare no competing interests.

\section{Additional information}

Supplementary information is available for this paper at https://doi.org/10.1038/s41467020-20089-0.

Correspondence and requests for materials should be addressed to J.-K.Z., J.D. or C.-G.D.

Peer review information Nature Communications thanks the anonymous reviewer(s) for their contribution to the peer review of this work. Peer review reports are available.

Reprints and permission information is available at http://www.nature.com/reprints

Publisher's note Springer Nature remains neutral with regard to jurisdictional claims in published maps and institutional affiliations.

(c) (i) Attribution 4.0 International License, which permits use, sharing, adaptation, distribution and reproduction in any medium or format, as long as you give appropriate credit to the original author(s) and the source, provide a link to the Creative Commons license, and indicate if changes were made. The images or other third party material in this article are included in the article's Creative Commons license, unless indicated otherwise in a credit line to the material. If material is not included in the article's Creative Commons license and your intended use is not permitted by statutory regulation or exceeds the permitted use, you will need to obtain permission directly from the copyright holder. To view a copy of this license, visit http://creativecommons.org/ licenses/by/4.0/.

(C) The Author(s) 2020 